\title{
Resonances of the Confined Hydrogen Atom and the Lamb-Dicke Effect in Non-Relativistic QED
}

\author{
Jérémy Faupin
}

\begin{abstract}
We study a model describing a system of one dynamical nucleus and one electron confined by their center of mass and interacting with the quantized electromagnetic field. We impose an ultraviolet cutoff and assume that the fine-structure constant is sufficiently small. Using a renormalization group method (based on $[3,4]$ ), we prove that the unperturbed eigenvalues turn into resonances when the nucleus and the electron are coupled to the radiation field. This analysis is related to the Lamb-Dicke effect.
\end{abstract}

\section{Introduction and statements of results}

\subsection{Introduction}

In this paper, we study a model of a hydrogen atom (or, more generally, a hydrogenoïd ion) confined by its center of mass. This model is used in theoretical physics to explain the Lamb-Dicke effect (see [7]); our purpose is to present, in the framework of non-relativistic quantum electrodynamics, a mathematically rigorous aspect of this phenomenon. Let us begin with describing it briefly.

First, consider a hydrogen atom whose nucleus is treated as static in quantum mechanics. The Schrödinger operator associated with it can be written as $-\Delta / 2 m_{1}+V$, where $m_{1}$ is the mass of the electron and $V$ is the Coulomb potential. If the electron is initially in some excited state of energy $E_{i}$, it can fall into a state of lower energy $E_{f}$ by spontaneous emission of a photon of energy

$$
|k|=E_{i}-E_{f} .
$$

Here $k$ is the momentum of the emitted photon and we have set $\hbar=c=1$, where $\hbar=h / 2 \pi, h$ is the Planck constant and $c$ is the velocity of light.

Next, consider a more realistic model where the nucleus is treated as dynamical. The internal energy of the atom is associated to the Schrödinger operator 
$-\Delta / 2 \mu+V$, where $\mu$ is the reduced mass, $\mu=m_{1} m_{2} /\left(m_{1}+m_{2}\right)$. We denote by $M=m_{1}+m_{2}$ the total mass of the system. Assume that the atom is initially in some excited state of internal energy $\left(m_{2} / M\right) E_{i}$. If the center of mass motion of the atomic system is free, then, as the internal state falls into a state of lower energy $\left(m_{2} / M\right) E_{f}$, the energy of the emitted photon is modified as follows:

$$
|k|=\frac{m_{2}}{M}\left(E_{i}-E_{f}\right)-\frac{k^{2}}{2 M}+\frac{k \cdot P}{M} .
$$

This identity comes from the conservation of both the energy and the momentum. Here $P$ denotes the momentum of the center of mass before the emission process. The term $k^{2} / 2 M$ corresponds to the recoil energy, whereas the term $k \cdot P / M$ is due to the Doppler effect.

Suppose finally that the center of mass of the atom is confined. Then the energy associated with its motion is quantized, as well as the internal energy. If $e_{i}$ and $e_{f}$ denote the energies associated with the center of mass motion respectively before and after the emission process, we have

$$
|k|=\frac{m_{2}}{M}\left(E_{i}-E_{f}\right)+e_{i}-e_{f} .
$$

In the Lamb-Dicke regime (in particular, for a sufficiently "strong" confinement), one can see that the most likely transitions are the ones such that $e_{i}=e_{f}$. In other words, in the scattering spectrum of the physical system, the most intense rays correspond exactly to the internal transitions of energies $\left(m_{2} / M\right)\left(E_{i}-E_{f}\right)$. These rays are, in addition, neither shifted by recoil effect nor broadened by Doppler effect. The suppression of both the recoil shift and the Doppler effect is then called the Lamb-Dicke effect.

This effect seems to have been discussed for the first time by R. H. Dicke in [9] where the author studies the reduction of the Doppler width of the light emitted by the molecules of a dense gas. It is assumed that the effect of collisions between the molecules of the gas is to confine their centers of mass. Then an emitter whose center of mass is trapped in a 1-dimensional square potential is considered.

Let us also mention that the Lamb-Dicke effect is frequently used in theoretical physics, for instance to study the cooling of atoms or ions by lasers (see, e.g., $[16,17])$. The idea of sideband laser cooling for an atom or an ion is indeed as follows: with the notations above, consider an ion in its ground state of energy $\left(m_{2} / M\right) E_{0}$. Let $\omega_{0}=\left(m_{2} / M\right)\left(E_{1}-E_{0}\right)$, and let $\gamma$ be the radiative line width of an excited state of energy $\left(m_{2} / M\right) E_{1}$. Assume that the ion is confined by a harmonic well of vibration frequency $\omega$; we suppose that $\omega \ll \gamma$, which insures in particular that the rays centered at the energies $\omega_{0} \pm(n+1 / 2) \omega$ are well-resolved. Assume furthermore that the external energy is initially equal to $e_{i}=(i+1 / 2) \omega$. The aim, to cool the ion, is to decrease this energy associated with the center of mass motion. To this end, a laser beam is tuned to a frequency $\omega_{L}=\omega_{0}-\omega$. The ion then "jumps" to a state of energy $E_{1}+e_{i-1}$, and is next likely to "fall" 
into a state of energy $E_{0}+e_{i-1}$, according to the Lamb-Dicke effect. This process is repeated, so that cooling proceeds, in principle, until the lowest vibrational energy $e_{0}$ is reached.

In $[6,7]$, the Lamb-Dicke effect is explained with the help of a Pauli-Fierz Hamiltonian describing a hydrogen atom confined by its center of mass. We shall consider the same model in this paper.

Mathematically, we shall have to face some difficulties in the spectral study of the Hamiltonian, because the electron and the nucleus themselves are not confined: they only interact with each other through the Coulomb potential. On the other hand, the confinement only exists in the direction of the center of mass. Thus one can imagine some states where the nucleus and the electron are localized very far from each other, and where, yet, the energy associated with the center of mass motion is low.

In [1], we obtained the existence of a ground state for the same model, for all values of the fine-structure constant. Now, as explained above, when instead of fixing the nucleus, it is only assumed that the center of mass of the atom or ion is confined, new intense rays appear in the scattering spectrum of the physical system. Thus, some resonances depending on the confining potential, with a very small imaginary part, should appear in the spectrum of the Hamiltonian. The aim of our paper is to prove this, assuming here that the fine-structure constant is sufficiently small.

In the standard model of non-relativistic quantum electrodynamics, the Hamiltonian that describes the system we consider is written as

$$
H_{U}^{V}=\sum_{j=1,2} \frac{1}{2 m_{j}}\left(p_{j}-q_{j} A\left(x_{j}\right)\right)^{2}+H_{f}+U(R)+V(r) .
$$

It acts on the Hilbert space $\mathrm{L}^{2}\left(\mathbb{R}^{6}\right) \otimes \mathcal{F}_{s}$, where $\mathcal{F}_{s}$ is the symmetric Fock space over $\mathrm{L}^{2}\left(\mathbb{R}^{3} \times \mathbb{Z}^{2}\right)$ for the transversal photons. The spins of the electron and of the nucleus are not taken into account. Here $x_{j}, q_{j}, p_{j}=-i \nabla_{j}, m_{j}$ denote respectively the position, the charge, the momentum and the mass of the particle $j$ (the electron or the nucleus). The position of the center of mass $R$ and the internal variable $r$ are defined by

$$
R:=\frac{m_{1} x_{1}+m_{2} x_{2}}{m_{1}+m_{2}}, \quad r:=x_{1}-x_{2} .
$$

Moreover, $A$ is the quantized electromagnetic vector potential in the Coulomb gauge, $H_{f}$ is the free photon energy field, $U$ is a confining potential acting on the center of mass of the atomic system, and $V$ denotes the Coulomb potential. An ultraviolet cutoff at a scale $\Lambda$ is imposed on $A$, for some arbitrary but finite $\Lambda>0$. Recall that the units have been chosen such that $\hbar=c=1$. We shall consider throughout the paper, for the sake of simplicity, a harmonic potential $U(R)=\beta^{2} R^{2}+c_{0}$, for some $\beta>0$ and $c_{0} \in \mathbb{R}$.

To address the problem of the existence of resonances, we follow the strategy developed in [3-5] and [2], based on a renormalization group analysis. In order to improve the infrared behavior of the interaction, as in [3], we shall transform $H_{U}^{V}$ 
through the usual Pauli-Fierz transformation. As a consequence, the interaction part in the transformed Hamiltonian can be treated as a perturbation growing in $x_{1}, x_{2}$. We shall use, to control the latter, the confining potential $U(R)$ together with a spatial cutoff $\chi(r)$ restricting the electron position to bounded distances from the nucleus position. Let $\widetilde{H}_{U}^{V}$ be the Hamiltonian obtained after the PauliFierz transformation and the spatial regularization.

The main result of this paper is Theorem 1 below which gives the existence of resonances for $\widetilde{H}_{U}^{V}$ as $q_{1}, q_{2}$ are $\neq 0$. Technically, the proof is significantly different from the one in $[3,4]$. Indeed, since a "part" of the $x_{1}, x_{2}$-behavior in the interaction will be controlled with the help of the confining potential $U(R)$, we shall have to use complex dilatations of both the photons and the nucleus-electron positions. Therefore we shall have to deal with non self-adjoint operators and non-orthogonal projections. We shall prove in Proposition 4 that the family of complex dilated Hamiltonians is analytic of type (A) in a neighborhood of the origin, which is not straightforward because of the presence of the confining potential. Besides, in the first step of the renormalization procedure, using $U(R)$ instead of introducing a cutoff $\chi(R)$ in the interaction requires estimates different from [3,4]. The main ones will be stated in Lemmas 9,10 and 14 . Our aim is to prove that $\widetilde{H}_{U}^{V}$ can be seen as a good starting point for the renormalization group analysis, referring next to [2] for the renormalization procedure itself. Thus we shall only reproduce the new aspects of the proofs, and refer otherwise to [3-5] or [2]. Note in addition that we will have to require a hypothesis related to the Fermi Golden Rule similar to the ones used in $[3,5]$; we will show in the appendix how to verify that it is satisfied.

The paper is organized as follows: in the remaining part of this section, we define our assumptions on the model we will work with, and we state our results. In Section 2, we study the Pauli-Fierz Hamiltonians $H_{U}^{V}$ and $\widetilde{H}_{U}^{V}$ associated with the model of the confined hydrogen atom. We prove in particular that $\theta \mapsto H_{U}^{V}(\theta)$ is analytic of type (A), which, by [5], leads to the absolute continuity of $\sigma\left(H_{U}^{V}\right)$ on an interval (Theorem 2). Next, in Section 3, we show how the renormalization group method developed in $[3,4]$ and [2] can be applied to our model. This gives the existence of resonances for $\widetilde{H}_{U}^{V}$ (Theorem 1). Finally as mentioned above, we verify in the appendix that a hypothesis related to the Fermi Golden Rule is satisfied.

\subsection{Assumptions on the model}

We shall work in this paper with more general operators than the Hamiltonians $H_{U}^{V}$ and $\widetilde{H}_{U}^{V}$ describing the model of the confined hydrogen atom. The operators we consider are supposed to act on $\mathrm{L}^{2}\left(\mathbb{R}^{6}\right) \otimes \mathcal{F}_{s}$ and are defined as

$$
H_{g}=H_{0}+W_{g}=H_{a t} \otimes \mathbf{1}+\mathbf{1} \otimes H_{f}+W_{g} .
$$

Here $H_{0}=H_{a t} \otimes \mathbf{1}+\mathbf{1} \otimes H_{f}$ denotes the unperturbed Hamiltonian. We assume that $H_{a t}$ is a Schrödinger operator on $\mathrm{L}^{2}\left(\mathbb{R}^{6}\right)$, such that its ground state energy $\lambda_{0,0}$ is strictly less than its ionization threshold $\Sigma:=\inf \sigma_{e s s}\left(H_{a t}\right)$, with at least one 
isolated eigenvalue located between $\lambda_{0,0}$ and $\Sigma$. We denote by $\left(\lambda_{j, 0}\right)$ the increasing sequence of eigenvalues of $H_{a t}$ located below $\Sigma$. The operator $H_{f}$ is defined by

$$
H_{f}=\int_{\underline{\mathbb{R}}^{3}}|k| a^{*}(\underline{k}) a(\underline{k}) d \underline{k} .
$$

Here we have used the notations

$$
\underline{\mathbb{R}}^{3}=\mathbb{R}^{3} \times \mathbb{Z}_{2}, \quad \underline{\mathbb{R}}^{3 n}=\left(\underline{\mathbb{R}}^{3}\right)^{n},
$$

and, for $\underline{k}=(k, \lambda) \in \underline{\mathbb{R}}^{3}$ and $a^{\#}=a^{*}$ or $a$,

$$
\int_{\underline{\mathbb{R}}^{3}} d \underline{k}=\sum_{\lambda=1,2} \int_{\mathbb{R}^{3}} d k, \quad a^{\#}(\underline{k})=a_{\lambda}^{\#}(k) .
$$

The usual creation operator $a_{\lambda}^{*}(k)$ and annihilation operator $a_{\lambda}(k)$ obey the following Canonical Commutation Rules (in the sense of operator-valued distributions):

$$
\begin{aligned}
& {\left[a_{\lambda}(k), a_{\lambda^{\prime}}^{*}\left(k^{\prime}\right)\right]=\delta_{\lambda \lambda^{\prime}} \delta\left(k-k^{\prime}\right),} \\
& {\left[a_{\lambda}(k), a_{\lambda^{\prime}}\left(k^{\prime}\right)\right]=\left[a_{\lambda}^{*}(k), a_{\lambda^{\prime}}^{*}\left(k^{\prime}\right)\right]=0 .}
\end{aligned}
$$

The interaction $W_{g}$ is written as

$$
W_{g}:=g W_{1}+g^{2} W_{2},
$$

with

$$
\begin{aligned}
W_{1}:= & \int_{\underline{\mathbb{R}}^{3}}\left[G_{1,0}(\underline{k}) \otimes a^{*}(\underline{k})+G_{0,1}(\underline{k}) \otimes a(\underline{k})\right] d \underline{k}, \\
W_{2}:= & \int_{\underline{\mathbb{R}}^{6}}\left[G_{2,0}\left(\underline{k}, \underline{k}^{\prime}\right) \otimes a^{*}(\underline{k}) a^{*}\left(\underline{k}^{\prime}\right)+G_{0,2}\left(\underline{k}, \underline{k}^{\prime}\right) \otimes a(\underline{k}) a\left(\underline{k}^{\prime}\right)\right. \\
& \left.+G_{1,1}\left(\underline{k}, \underline{k}^{\prime}\right) \otimes a^{*}(\underline{k}) a\left(\underline{k}^{\prime}\right)\right] d \underline{k} d \underline{k}^{\prime} .
\end{aligned}
$$

We assume that, for all $\underline{k}, \underline{k}^{\prime}, G_{m, n}\left(\underline{k}, \underline{k}^{\prime}\right)$ defines an operator on $\mathrm{L}^{2}\left(\mathbb{R}^{6}\right)$. We will sometimes use the notations $W_{1,0}:=\int G_{1,0}(\underline{k}) \otimes a^{*}(\underline{k}) d \underline{k}$, and similarly for the other operators $W_{m, n}, m+n \in\{1,2\}$.

As in [5], we shall dilate both the positions $x_{j}$ of the two particles (the electron and the nucleus) and the momenta $k$ of the photons in the following way:

$$
x_{j} \mapsto e^{\theta} x_{j}, \quad k \mapsto e^{-\theta} k .
$$

We denote by $H_{a t}(\theta), W_{g}(\theta), G_{m, n}(\theta)$ and $H_{g}(\theta)$ the families of operators obtained respectively from $H_{a t}, W_{g}, G_{m, n}$ and $H_{g}$ through this complex scaling. We shall assume throughout the paper that $G_{m, n}(\theta)^{*}=G_{n, m}(\bar{\theta})$. In addition we set

$$
\left(\mathcal{H}_{a n}\right)\left\{\begin{array}{l}
\text { There exists } \theta_{0}>0 \text { such that for all } \theta \text { in the disc } D\left(0, \theta_{0}\right), \\
\left\|\left[H_{a t}(\theta)-H_{a t}\right]\left[H_{a t}+i\right]^{-1}\right\| \leq b\left(\theta_{0}\right) \text {, where } b\left(\theta_{0}\right) \rightarrow 0 \text { as } \theta_{0} \rightarrow 0 . \\
\text { Moreover, the map } \theta \mapsto H_{g}(\theta) \text { is analytic of type }(\mathrm{A}) \text { on } D\left(0, \theta_{0}\right) .
\end{array}\right.
$$


As for the interaction, we require the following hypotheses:

$$
\left(\mathcal{H}_{-1 / 2}\right)\left\{\begin{array}{l}
\text { There exist a non-negative function } J_{-1 / 2}(k) \text { and } \theta_{0}>0 \text { such } \\
\text { that: } \\
\text { (i) } \sup _{|\theta| \leq \theta_{0}}\left\|G_{m, n}(\underline{k} ; \theta)\left|H_{a t}+i\right|^{-1 / 2}\right\| \leq J_{-1 / 2}(k) \text { for } m+n=1, \\
\text { (ii) } \sup _{|\theta| \leq \theta_{0}}\left\|G_{m, n}\left(\underline{k}, \underline{k}^{\prime} ; \theta\right)\right\| \leq J_{-1 / 2}(k) J_{-1 / 2}\left(k^{\prime}\right) \text { for } m+n=2, \\
\text { (iii) } \int_{\mathbb{R}^{3}}\left(1+|k|^{-1}\right) J_{-1 / 2}(k)^{2} d k<\infty .
\end{array}\right.
$$

Moreover, the maps $\theta \mapsto G_{m, n}\left(\underline{k}, \underline{k}^{\prime} ; \theta\right)$ are bounded analytic on $D\left(0, \theta_{0}\right)$ with respect to the norms given in (i) and (ii) respectively.

A similar assumption is required in $[3,4]$. Here we need another assumption related to the confinement of the center of mass imposed in our model:

$$
\left(\mathcal{H}_{1 / 2}\right)\left\{\begin{aligned}
& \text { There exist a non-negative function } J_{1 / 2}(k) \text { and } \theta_{0}>0 \text { such that: } \\
& \text { (i) } \sup \left\|G_{m, n}(\underline{k} ; \theta)\left|H_{a t}+i\right|^{-1}\right\| \leq J_{1 / 2}(k) \text { for } m+n=1, \\
& \text { (ii) } \quad \sup \left\|G_{m, n}\left(\underline{k}, \underline{k^{\prime}} ; \theta\right)\left|H_{a t}+i\right|^{-1}\right\| \leq J_{1 / 2}(k) J_{1 / 2}\left(k^{\prime}\right), \\
& \sup \left\|G_{m, n}\left(\underline{k}, \underline{k}^{\prime} ; \theta\right)\left|H_{a t}+i\right|^{-1 / 2}\right\| \leq J_{1 / 2}(k) J_{-1 / 2}\left(k^{\prime}\right), \\
& \quad \text { for } m+n=2, \text { where } J_{-1 / 2} \text { is defined in Hypothesis } \\
&\left(\mathcal{H}_{-1 / 2}\right), \\
& \text { (iii) } \sup _{k \in \mathbb{R}^{3}}\left\{|k|^{\frac{1}{2}(1-\mu)} J_{1 / 2}(k)\right\}<\infty \text { for some } \mu>0 .
\end{aligned}\right.
$$

Note that in Hypothesis $\left(\mathcal{H}_{1 / 2}\right)$, it is implicitly assumed that $\left(\mathcal{H}_{-1 / 2}\right)$ is satisfied and that for $m+n=2, G_{m, n}$ is symmetric under the permutation of the variables $\underline{k}$ and $\underline{k}^{\prime}$.

\subsection{Statement of results}

Our main result is Theorem 1 below which provides the existence of resonances for $H_{g}$ under Hypotheses $\left(\mathcal{H}_{a n}\right),\left(\mathcal{H}_{-1 / 2}\right)$ and $\left(\mathcal{H}_{1 / 2}\right)$. More precisely, considering an eigenvalue $\lambda_{j, 0}$ of $H_{0}$, we shall prove that there exist resonances $\lambda_{j, g}$ of $H_{g}$, such that

$$
\lambda_{j, g} \underset{g \rightarrow 0}{\rightarrow} \lambda_{j, 0} .
$$

We follow the strategy of [3,4] and use the smooth Feshbach map defined in [2]. The proof is not straightforward since, as explained above, we have to modify carefully Hypotheses 2 and 3 stated in $[3,4]$ in such a way that, on one hand, our new hypotheses are well adapted to our model, and on the other hand, they are still sufficient to perform a renormalization group analysis. This is reflected in our choice of Hypotheses $\left(\mathcal{H}_{-1 / 2}\right)$ and $\left(\mathcal{H}_{1 / 2}\right)$ above. We shall prove: 
Theorem 1. Let $\lambda_{j, 0}$ be a non-degenerate eigenvalue of $H_{0}$ such that $\lambda_{0,0}<\lambda_{j, 0}<\Sigma$. Let the coupling parameter $g>0$ be sufficiently small. Assume that Hypotheses $\left(\mathcal{H}_{a n}\right),\left(\mathcal{H}_{-1 / 2}\right)$ and $\left(\mathcal{H}_{1 / 2}\right)$ hold for some $\theta_{0}>0$ sufficiently small. Let $\delta_{j}$ be the distance from $\lambda_{j, 0}$ to the rest of the spectrum of $H_{a t}$. Pick $\theta=\eta+i \nu$ in the disc $D\left(0, \theta_{0}\right)$ and $\rho_{0}>0$ sufficiently small, such that $\rho_{0} \leq\left(\delta_{j} \sin \nu\right) / 2<1$. Then the spectrum of $H_{g}(\theta)$ in $D_{\rho_{0} / 2}:=D\left(\lambda_{j, 0}, \rho_{0} / 2\right)$ is located as follows:

$$
\sigma\left(H_{g}(\theta)\right) \cap D_{\rho_{0} / 2} \subset \lambda_{j, g}(\theta)+K_{l, n}(\theta),
$$

where $\lambda_{j, g}(\theta)$ is a non-degenerate eigenvalue of $H_{g}(\theta)$, and where $K_{l, n}(\theta)$ is a complex domain defined, for some $\tau>1$ and $0<\mathrm{C}<1$, by:

$$
K_{l, n}(\theta):=\left\{\lambda_{j, g}(\theta)+e^{-i \nu} a+b, 0 \leq a \leq 1,|b| \leq \mathrm{C} a^{\tau}\right\} .
$$

In particular, $\lambda_{j, g}(\theta)$ is independent of $\theta$.

Thus, as stated at the beginning of this subsection, provided that Hypothesis $\left(\mathcal{H}_{\Gamma_{j}}\right)$ (see Subsection 3.1) related to the Fermi golden rule holds, the unperturbed eigenvalue $\lambda_{j, 0}$ turns into a resonance when the nucleus and the electron are coupled to the photons.

If Hypothesis $\left(\mathcal{H}_{1 / 2}\right)$ is not satisfied, following [5], we still have:

Theorem 2. Let $g>0$ sufficiently small. Assume that Hypotheses $\left(\mathcal{H}_{\text {at }}\right)$ and $\left(\mathcal{H}_{-1 / 2}\right)$ hold for some $\theta_{0}>0$ sufficiently small. Then, provided that Hypotheses $\left(\mathcal{H}_{\Gamma_{j}}\right)$ (see Subsection 3.1) hold for all non-perturbed eigenvalues $\lambda_{j, 0}$ located below the ionization threshold $\Sigma$ of $H_{a t}$, the spectrum of $H_{g}$ is absolutely continuous on $\left[E_{g}, \Sigma\right] \backslash \mathcal{V}$, where $E_{g}$ denotes the ground state energy of $H_{g}$ and $\mathcal{V}$ is a neighborhood of order $O\left(g^{2}\right)$ of $\left\{E_{g}\right\} \cup\{\Sigma\}$.

The proof of this result follows from the existence of a Feshbach operator associated with $H_{g}(\theta)$; this can be obtained from Lemmas 9 and 10 instead of using the method of [5].

Finally, in the next section, we shall prove that Hypotheses $\left(\mathcal{H}_{a n}\right),\left(\mathcal{H}_{-1 / 2}\right)$ and $\left(\mathcal{H}_{1 / 2}\right)$ are well-adapted to the model of the confined hydrogen atom, that is

1. The initial Hamiltonian $H_{U}^{V}$ fulfills Hypotheses $\left(\mathcal{H}_{a n}\right)$ and $\left(\mathcal{H}_{-1 / 2}\right)$.

2. The regularized Hamiltonian $\widetilde{H}_{U}^{V}$ fulfills Hypotheses $\left(\mathcal{H}_{a n}\right),\left(\mathcal{H}_{-1 / 2}\right)$ and $\left(\mathcal{H}_{1 / 2}\right)$.

\section{The model of the confined hydrogen atom}

\subsection{Definition of the Hamiltonian $H_{U}^{V}$}

Recall that the Hamiltonian $H_{U}^{V}$ we want to study is written as

$$
H_{U}^{V}:=\sum_{j=1,2} \frac{1}{2 m_{j}}\left(p_{j}-q_{j} A_{j}\right)^{2}+H_{f}+U+V .
$$




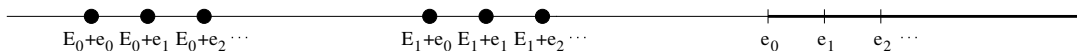

FiguRE 1. Spectrum of the atomic Hamiltonian $H_{a t}$.

It acts on the Hilbert space $\mathrm{L}^{2}\left(\mathbb{R}^{6}\right) \otimes \mathcal{F}_{s} \simeq \mathrm{L}^{2}\left(\mathbb{R}^{6} ; \mathcal{F}_{s}\right)$, where $\mathcal{F}_{s}$ denotes the symmetric Fock space of transversally polarized photons over $\mathrm{L}^{2}\left(\underline{\mathbb{R}}^{3}\right)$, that is

$$
\mathcal{F}_{s}:=\mathcal{F}_{s}\left(\mathrm{~L}^{2}\left(\underline{\mathbb{R}}^{3}\right)\right)=\mathbb{C} \oplus \bigoplus_{n \geq 1} S_{n} \otimes_{k=1}^{n} \mathrm{~L}^{2}\left(\underline{\mathbb{R}}^{3}\right) .
$$

Here $S_{n}$ denotes the symmetrization of the $n$ components in the tensor product $\otimes_{k=1}^{n} \mathrm{~L}^{2}\left(\underline{\mathbb{R}}^{3}\right)$. The vector potential $A_{j}$ in the Coulomb gauge is defined by

$$
A_{j}:=\int_{\mathbb{R}^{6}}^{\oplus} A\left(x_{j}\right) d X
$$

with $X=\left(x_{1}, x_{2}\right)$ and

$$
A(x):=\frac{1}{2 \pi} \sum_{\lambda=1,2} \int_{\mathbb{R}^{3}} \frac{\widehat{\chi}_{\Lambda}(k)}{\sqrt{|k|}} \varepsilon_{\lambda}(k)\left(a_{\lambda}^{*}(k) e^{-i k \cdot x}+a_{\lambda}(k) e^{i k \cdot x}\right) d k .
$$

Here $\widehat{\chi}_{\Lambda}$ denotes an ultraviolet cutoff function. Some analyticity of $\widehat{\chi}_{\Lambda}$ is required, so that we choose:

$$
\widehat{\chi}_{\Lambda}(k)=e^{-k^{2} /\left(Z^{4} q^{8} \Lambda^{2}\right)} .
$$

Besides, in $(21), \varepsilon_{1}(k)$ and $\varepsilon_{2}(k)$ denote polarization vectors that are perpendicular to each other and to $k$. The free field energy operator $H_{f}$ is defined in (7). We write the Coulomb potential $V$ as

$$
V(r):=-Z q^{2} \frac{\mathrm{C}}{|r|},
$$

where $\mathrm{C}$ is a positive constant. We set

$$
P:=p_{1}+p_{2}, \quad \frac{p}{\mu}:=\frac{p_{1}}{m_{1}}-\frac{p_{2}}{m_{2}} .
$$

The atomic Hamiltonian is then given in this case by

$$
H_{a t}=\sum_{j=1,2} \frac{p_{j}^{2}}{2 m_{j}}+U+V \simeq\left(\frac{p^{2}}{2 \mu}+V\right) \otimes \mathbf{1}+\mathbf{1} \otimes\left(\frac{P^{2}}{2 M}+U\right) .
$$

We denote by $\left(E_{j}\right)_{j \geq 0}$ the non-decreasing sequence of eigenvalues of $p^{2} / 2 \mu+V$ and by $\left(e_{j}\right)_{j \geq 0}$ the non-decreasing sequence of eigenvalues of $P^{2} / 2 M+U$. The spectrum of $H_{a t}$ is pictured in Figure 1.

As in [3], we proceed to a change of units in order to exhibit the perturbative character of the problem. More precisely, we consider the unitary operator $\mathcal{U}_{1}$ that dilates the electron and nucleus positions, and the photons momenta, through $\left(x_{j}, k\right) \mapsto\left(x_{j} / Z q^{2}, Z^{2} q^{4} k\right)$. This leads to

$$
\frac{1}{Z^{2} q^{4}} \mathcal{U}_{1} H_{U}^{V} \mathcal{U}_{1}^{*}=\sum_{j=1,2} \frac{1}{2 m_{j}}\left(p_{j}-q_{j} Z q^{2} \widetilde{A}_{j}\left(Z q^{2} \cdot\right)\right)^{2}+H_{f}+\widetilde{V}+\widetilde{U}
$$




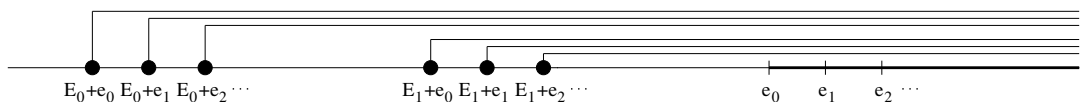

Figure 2. Spectrum of the unperturbed Hamiltonian $H_{0}$.

The vector potential $\widetilde{A}(x)$ denotes $A(x)$ where $\widehat{\chi}_{\Lambda}$ is replaced by $\widehat{\chi}_{\Lambda}\left(Z^{2} q^{4} \cdot\right)$. Moreover we have set $\widetilde{V}(r):=-\mathrm{C} /|r|$ and $\widetilde{U}(R):=\left(1 / Z^{2} q^{4}\right) U\left(R / Z q^{2}\right)$. We redefine $\widehat{\chi}_{\Lambda}(k):=\widehat{\chi}_{\Lambda}\left(Z^{2} q^{4} k\right), V(r):=\widetilde{V}(r)$ and $U(R):=\widetilde{U}(R)$. Thus the new Hamiltonian, still denoted by $H_{U}^{V}$, that we have to consider, is

$$
H_{U}^{V}=\sum_{j=1,2} \frac{1}{2 m_{j}}\left(p_{j}-q_{j} Z q^{2} A_{j}\left(Z q^{2} \cdot\right)\right)^{2}+H_{f}+U+V .
$$

Setting $g:=\left(q^{2} \Lambda\right)^{3 / 2}$, we can write $H_{U}^{V}$ as $H_{U}^{V}:=H_{0}+W_{g}+\Lambda_{0}$, where $H_{0}$ is defined by

$$
H_{0}:=\sum_{j=1,2} \frac{p_{j}^{2}}{2 m_{j}}+U+V+H_{f}=H_{a t} \otimes \mathbf{1}+\mathbf{1} \otimes H_{f}
$$

Since the spectrum of $H_{f}$ consists of the simple eigenvalue 0 , and the half-axis ] $0 ; \infty\left[\right.$ as absolutely continuous spectrum, we obtain $\sigma\left(H_{0}\right)$ as pictured in Figure 2.

We write the interaction $W_{g}$ as in (11)-(13), where the operators $G_{m, n}:=$ $\sum_{j=1,2} G_{m, n}^{j}$ are given by

$$
\begin{aligned}
G_{1,0}^{1}(\underline{k}) & =G_{0,1}^{1}(\underline{k})^{*}=\frac{i Z}{2 m_{1} \Lambda^{3 / 2}} \frac{\widehat{\chi}_{\Lambda}(k)}{2 \pi \sqrt{|k|}} e^{-i Z q^{2} k \cdot x_{1}} \varepsilon_{\lambda}(k) \cdot \nabla_{x_{1}}, \\
G_{1,0}^{2}(\underline{k}) & =G_{0,1}^{2}(\underline{k})^{*}=\frac{-i Z^{2}}{2 m_{2} \Lambda^{3 / 2}} \frac{\widehat{\chi}_{\Lambda}(k)}{2 \pi \sqrt{|k|}} e^{-i Z q^{2} k \cdot x_{2}} \varepsilon_{\lambda}(k) \cdot \nabla_{x_{2}}, \\
G_{2,0}^{1}\left(\underline{k}, \underline{k}^{\prime}\right) & =G_{0,2}^{1}\left(\underline{k}, \underline{k}^{\prime}\right)^{*} \\
& =\frac{Z^{2}}{2 m_{1} \Lambda^{3}} \frac{\widehat{\chi}_{\Lambda}(k) \widehat{\chi}_{\Lambda}\left(k^{\prime}\right)}{4 \pi^{2} \sqrt{|k|\left|k^{\prime}\right|}} \varepsilon_{\lambda}(k) \cdot \varepsilon_{\lambda^{\prime}}\left(k^{\prime}\right) e^{-i Z q^{2} k \cdot x_{1}} e^{-i Z q^{2} k^{\prime} \cdot x_{1},} \\
G_{2,0}^{2}\left(\underline{k}, \underline{k}^{\prime}\right) & =G_{0,2}^{2}\left(\underline{k}, \underline{k}^{\prime}\right)^{*} \\
& =\frac{Z^{4}}{2 m_{2} \Lambda^{3}} \frac{\widehat{\chi}_{\Lambda}(k) \widehat{\chi}_{\Lambda}\left(k^{\prime}\right)}{4 \pi^{2} \sqrt{|k|\left|k^{\prime}\right|}} \varepsilon_{\lambda}(k) \cdot \varepsilon_{\lambda^{\prime}}\left(k^{\prime}\right) e^{-i Z q^{2} k \cdot x_{2}} e^{-i Z q^{2} k^{\prime} \cdot x_{2},} \\
G_{1,1}^{1}\left(\underline{k}, \underline{k}^{\prime}\right) & =\frac{Z^{2}}{2 m_{1} \Lambda^{3}} \frac{\widehat{\chi}_{\Lambda}(k) \widehat{\chi}_{\Lambda}\left(k^{\prime}\right)}{2 \pi^{2} \sqrt{|k|\left|k^{\prime}\right|}} \varepsilon_{\lambda}(k) \cdot \varepsilon_{\lambda^{\prime}}\left(k^{\prime}\right) e^{-i Z q^{2} k \cdot x_{1}} e^{i Z q^{2} k^{\prime} \cdot x_{1}}, \\
G_{1,1}^{2}\left(\underline{k}, \underline{k}^{\prime}\right) & =\frac{Z^{4}}{2 m_{2} \Lambda^{3}} \frac{\widehat{\chi}_{\Lambda}(k) \widehat{\chi}_{\Lambda}\left(k^{\prime}\right)}{2 \pi^{2} \sqrt{|k|\left|k^{\prime}\right|}} \varepsilon_{\lambda}(k) \cdot \varepsilon_{\lambda^{\prime}}\left(k^{\prime}\right) e^{-i Z q^{2} k \cdot x_{2}} e^{i Z q^{2} k^{\prime} \cdot x_{2}} .
\end{aligned}
$$


Finally the real number $\Lambda_{0}$ is defined by $\Lambda_{0}:=\sum_{j=1,2} \Lambda_{0}^{j}$ where

$$
\Lambda_{0}^{1}=\frac{Z^{2}}{4 \pi^{2} m_{1} \Lambda^{3}} \int_{\mathbb{R}^{3}} \frac{\widehat{\chi}_{\Lambda}(k)}{|k|} d k, \quad \Lambda_{0}^{2}=\frac{Z^{4}}{4 \pi^{2} m_{2} \Lambda^{3}} \int_{\mathbb{R}^{3}} \frac{\widehat{\chi}_{\Lambda}(k)}{|k|} d k .
$$

Henceforth we remove this constant from the interaction by redefining $H_{0}:=$ $H_{0}+\Lambda_{0}, W_{g}:=W_{g}-\Lambda_{0}$.

\subsection{Analyticity of $H_{U}^{V}(\theta)$}

In this paper, in the same way as in [5], we scale both the electron-nucleus positions and the photons momenta through

$$
x_{j} \mapsto e^{\theta} x_{j}, \quad k \mapsto e^{-\theta} k .
$$

The scaling parameter $\theta$ is assumed to lie in a disc $D\left(0, \theta_{0}\right) \subset \mathbb{C}$. For real $\theta$, the transformations $(33)$ determine a unitary operator $\mathcal{U}_{\theta}$ such that:

$$
\begin{aligned}
* \mathcal{U}_{\theta}\left(\frac{p^{2}}{2 \mu}+V\right) \mathcal{U}_{\theta}^{*} & =e^{-2 \theta} \frac{p^{2}}{2 \mu}+e^{-\theta} V, \\
* \mathcal{U}_{\theta}\left(\frac{P^{2}}{2 M}+U\right) \mathcal{U}_{\theta}^{*} & =e^{-2 \theta} \frac{P^{2}}{2 M}+U\left(e^{\theta} \cdot\right), \\
* \quad \mathcal{U}_{\theta} H_{f} \mathcal{U}_{\theta}^{*} & =e^{-\theta} H_{f} .
\end{aligned}
$$

Recall that $U(R)=\beta^{2} R^{2}+\mathrm{c}_{0}$. For $\theta \in D\left(0, \theta_{0}\right)$, we define the quadratic forms $q_{V}^{\theta}$ and $q_{U}^{\theta}$ on $\mathrm{H}^{1}\left(\mathbb{R}^{3}\right)$ and $\mathrm{H}^{1}\left(\mathbb{R}^{3}\right) \cap Q\left(U^{+}\right)$respectively by

$$
\begin{aligned}
q_{V}^{\theta}(\phi, \psi) & :=\frac{e^{-2 \theta}}{2 \mu}(p \phi, p \psi)-e^{-\theta}\left(\left(V^{-}\right)^{1 / 2} \phi,\left(V^{-}\right)^{1 / 2} \psi\right), \\
q_{U}^{\theta}(\phi, \psi) & :=\frac{e^{-2 \theta}}{2 M}(P \phi, P \psi)+e^{2 \theta} \beta^{2}(R \phi, R \psi)+\mathrm{c}_{0}(\phi, \psi) .
\end{aligned}
$$

Lemma 3. Let $\theta_{0}$ be sufficiently small. For $\theta$ in $D\left(0, \theta_{0}\right)$, let $H_{0}(\theta)$ be the operator associated with the strictly $m$-sectorial quadratic form

$$
q_{H_{0}}^{\theta}(\Phi, \Psi)=q_{V}^{\theta}(\Phi, \Psi)+q_{U}^{\theta}(\Phi, \Psi)+e^{-\theta}\left(H_{f}^{1 / 2} \Phi, H_{f}^{1 / 2} \Psi\right),
$$

on $Q\left(p_{1}^{2}+p_{2}^{2}\right) \cap Q\left(U^{+}\right) \cap Q\left(H_{f}\right)$. Then $\theta \mapsto H_{0}(\theta)$ is analytic of type $(A)$ on $D\left(0, \theta_{0}\right)$ and

$$
\sigma\left(H_{0}(\theta)\right)=\sigma\left(e^{-2 \theta} p^{2} / 2 \mu+e^{-\theta} V\right)+\sigma\left(e^{-2 \theta} P^{2} / 2 M+U\left(e^{\theta} \cdot\right)\right)+\sigma\left(e^{-\theta} H_{f}\right),
$$

where $e^{-2 \theta} p^{2} / 2 \mu+e^{-\theta} V$ and $e^{-2 \theta} P^{2} / 2 M+U\left(e^{\theta} \cdot\right)$ are the operators associated respectively with the strictly $m$-sectorial quadratic forms $q_{V}^{\theta}$ and $q_{U}^{\theta}$.

Proof. Fix $\theta$ in $D\left(0, \theta_{0}\right)$ for a sufficiently small $\theta_{0}$. It is easy to see that the quadratic forms $q_{V}^{\theta}$ and $q_{U}^{\theta}$ defined in (35)-(36) are strictly m-sectorial. Applying Ichinose's lemma (see [14]), we obtain that $q_{H_{0}}^{\theta}$ is also strictly m-sectorial and 


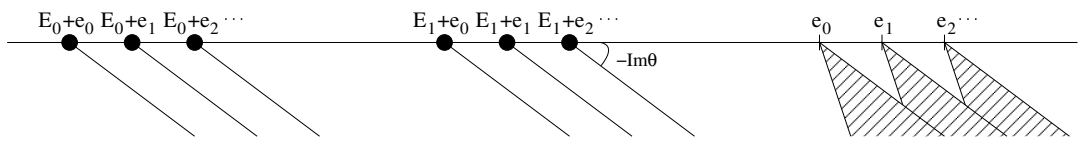

Figure 3. Spectrum of the complex dilated unperturbed Hamiltonian $H_{0}(\theta)$.

that (38) is satisfied. It remains to prove analyticity of type (A). Let $\Phi \in D\left(H_{0}\right)$. For all $\Psi \in D\left(H_{0}(\theta)^{*}\right)$, we have

$$
\begin{aligned}
\left|\left(H_{0}(\theta)^{*} \Psi, \Phi\right)\right| & \leq\left|\left(\Psi, H_{0} \Phi\right)\right|+\left|q_{H_{0}}^{\theta}(\Psi, \Phi)-q_{H_{0}}(\Psi, \Phi)\right| \\
& \leq b\left(\theta_{0}\right)\|\Psi\|\left(\left\|p^{2} \Phi\right\|+\|V \Phi\|+\left\|P^{2} \Phi\right\|+\left\|R^{2} \Phi\right\|+\left\|H_{f} \Phi\right\|\right),
\end{aligned}
$$

where $b\left(\theta_{0}\right)$ is such that $b\left(\theta_{0}\right) \rightarrow 0$ as $\theta_{0} \rightarrow 0$. It follows that $D\left(H_{0}\right) \subset D\left(H_{0}(\theta)\right)$. Then, from $\left\|\left[H_{0}-H_{0}(\theta)\right]\left[H_{0}+i\right]^{-1}\right\| \leq b\left(\theta_{0}\right)$, we get

$$
\left\|H_{0} \Phi\right\| \leq \frac{1}{1-b\left(\theta_{0}\right)}\left(\left\|H_{0}(\theta) \Phi\right\|+b\left(\theta_{0}\right)\|\Phi\|\right),
$$

for all $\Phi \in D\left(H_{0}\right)$, provided that $b\left(\theta_{0}\right)<1$. This implies that $H_{0}(\theta)$ is closed on $D\left(H_{0}\right)$ and hence that $D\left(H_{0}\right)=D\left(H_{0}(\theta)\right)$. The analyticity of the map $\theta \mapsto H_{0}(\theta) \Phi$ for $\Phi \in D\left(H_{0}\right)$ is then straightforward.

According to (38), the spectrum of $H_{0}(\theta)$ is given by Figure 3 .

Now, the operator $\mathcal{U}_{\theta} W_{g} \mathcal{U}_{\theta}^{*}$ is $W_{g}$ where the operator-valued functions $G_{m, n}^{j}$ are replaced by $G_{m, n}^{j}(\theta)$; we get $G_{m, n}^{j}(\theta)$ by adding a factor $e^{-2 \theta}$ and replacing $\widehat{\chi}_{\Lambda}(k)$ by $\widehat{\chi}_{\Lambda}\left(e^{-\theta} k\right)$ in $G_{m, n}^{j}$. For instance

$$
G_{1,0}^{1}(\underline{k} ; \theta)=G_{0,1}^{1}(\underline{k} ; \bar{\theta})^{*}=e^{-2 \theta} \frac{i Z}{2 m_{1} \Lambda^{3 / 2}} \frac{\widehat{\chi}_{\Lambda}\left(e^{-\theta} k\right)}{2 \pi \sqrt{|k|}} e^{-i Z q^{2} k \cdot x_{1}} \varepsilon_{\lambda}(k) \cdot \nabla_{x_{1}} .
$$

The operator $W_{g}(\theta)$ is well-defined on $D\left(H_{0}\right)$.

Proposition 4. Let $\theta_{0}$ and $g$ be sufficiently small. For $\theta$ in $D\left(0, \theta_{0}\right)$, let $H_{U}^{V}(\theta):=$ $H_{0}(\theta)+W_{g}(\theta)$ on $D\left(H_{0}\right)$. Then $H_{U}^{V}(\theta)$ fulfills Hypotheses $\left(\mathcal{H}_{a n}\right)$ and $\left(\mathcal{H}_{-1 / 2}\right)$, with

$$
J_{-1 / 2}(k) \leq \text { Cste }|k|^{-1 / 2} e^{-k^{2} / \Lambda^{2}} .
$$

Proof. The fact that $\left\|\left[H_{a t}(\theta)-H_{a t}\right]\left[H_{a t}+i\right]^{-1}\right\| \leq b\left(\theta_{0}\right)$, for some $b\left(\theta_{0}\right)$ such that $b\left(\theta_{0}\right) \rightarrow 0$ as $\theta_{0} \rightarrow 0$, follows in the same way as in the proof of Lemma 3. As in [5, Lemma 1.1], one can see that $\left\|W_{g}(\theta)\left[H_{0}+i\right]^{-1}\right\| \leq \mathrm{C} g$, for some positive constant C. This implies that $H_{U}^{V}(\theta)$ is closed on $D\left(H_{0}\right)$ for $g$ and $\theta_{0}$ sufficiently small. Since $\theta \mapsto W_{g}(\theta)\left[H_{0}+i\right]^{-1}$ is analytic on $D\left(0, \theta_{0}\right)$ by our choice (22) of the ultraviolet cutoff, the analyticity of type (A) of $\theta \mapsto H_{U}^{V}(\theta)$ follows from Lemma 3. Finally, using the definition of $G_{m, n}(\theta)$ given in (29)-(31) and (41), one can straightforwardly prove that $H_{U}^{V}(\theta)$ fulfills Hypothesis $\left(\mathcal{H}_{-1 / 2}\right)$ with $J_{-1 / 2}$ given by (42). 


\subsection{The regularized Hamiltonian $\widetilde{H}_{U}^{V}$}

We proved in Proposition 4 that $H_{U}^{V}(\theta)$ fulfills Hypothesis $\left(\mathcal{H}_{-1 / 2}\right)$. This will be sufficient to obtain the absolute continuity of $\sigma\left(H_{U}^{V}\right)$ on an interval between $\lambda_{0,0}$ and $\Sigma$, but this is not sufficient to apply the renormalization group method of $[3,4]$. To face this problem, we begin with performing the Power-Zienau-Woolley transformation (sometimes called the Pauli-Fierz transformation) on $H_{U}^{V}$. More precisely, setting $X=\left(x_{1}, x_{2}\right)$, we define a unitary operator $\mathcal{T}$ by

$$
\mathcal{T}=\int_{\mathbb{R}^{6}}^{\oplus} \mathcal{T}(X) d X \quad \text { with } \quad \mathcal{T}(X)=e^{-i \sum_{j=1,2} q_{j} Z q^{2} x_{j} \cdot A(0)} .
$$

Then $b_{\lambda}(k, X):=\mathcal{T}(X) a_{\lambda}(k) \mathcal{T}^{*}(X)=a_{\lambda}(k)-i w_{\lambda}(k, X)$, with

$$
w_{\lambda}(k, X)=\frac{1}{2 \pi} \frac{\widehat{\chi}_{\Lambda}(k)}{|k|^{1 / 2}} \varepsilon_{\lambda}(k) \cdot \sum_{j=1,2} q_{j} Z q^{2} x_{j} .
$$

The Hamiltonian $\widetilde{H}_{U}^{V}$, which is unitary equivalent to $H_{U}^{V}$, is defined by

$$
\widetilde{H}_{U}^{V}:=\mathcal{T} H_{U}^{V} \mathcal{T}^{*}=\sum_{j=1,2} \frac{1}{2 m_{j}}\left(p_{j}-q_{j} Z q^{2} \widetilde{A}_{j}\left(Z q^{2} \cdot\right)\right)^{2}+\widetilde{H}_{f}+U+V
$$

with $\widetilde{A}_{j}=\int_{\mathbb{R}^{6}}^{\oplus} \widetilde{A}_{j}(X) d X, \widetilde{H}_{f}=\int_{\mathbb{R}^{6}}^{\oplus} \widetilde{H}_{f}(X) d X$, and

$$
\widetilde{A}_{j}(X)=A\left(x_{j}\right)-A(0), \quad \widetilde{H}_{f}(X)=\sum_{\lambda=1,2} \int_{\mathbb{R}^{3}}|k| b_{\lambda}^{*}(k, X) b_{\lambda}(k, X) d k .
$$

Expanding (45), we can write

$$
\widetilde{H}_{U}^{V}:=\widetilde{H}_{0}+\widetilde{W}_{g}:=\widetilde{H}_{a t}+H_{f}+\widetilde{W}_{g}:=\widetilde{H}_{a t}+H_{f}+g \widetilde{W}_{1}+g^{2} \widetilde{W}_{2},
$$

with

$$
\begin{aligned}
\widetilde{H}_{a t}= & H_{a t}+g^{2} \frac{Z^{2}}{\Lambda^{3}} \sum_{\lambda=1,2} \int_{\mathbb{R}^{3}} \frac{\hat{\chi}_{\Lambda}(k)^{2}}{4 \pi^{2}}\left(\varepsilon_{\lambda}(k) \cdot \widetilde{r}\right)^{2} d k \\
& +2 g^{2} \frac{Z^{2}}{\Lambda^{3}} \int_{\mathbb{R}^{3}} \frac{\widehat{\chi}_{\Lambda}(k)^{2}}{\pi^{2}|k|}\left[\frac{1}{2 m_{1}} \sin ^{2}\left(Z q^{2} k \cdot x_{1} / 2\right)+\frac{Z}{2 m_{2}} \sin ^{2}\left(Z q^{2} k \cdot x_{2} / 2\right)\right] d k,
\end{aligned}
$$

and

$$
\widetilde{W}_{1}=\int_{\underline{\mathbb{R}}^{3}}\left[\widetilde{G}_{1,0}(\underline{k}) \otimes a^{*}(\underline{k})+\widetilde{G}_{0,1}(\underline{k}) \otimes a(\underline{k})\right] d \underline{k} .
$$


The "new" coupling operators $\widetilde{G}_{m, n}$ are defined for $m+n=1$ by

$$
\begin{aligned}
\widetilde{G}_{1,0}(\underline{k})= & \widetilde{G}_{0,1}(\underline{k})^{*} \\
= & \frac{i Z}{2 m_{1} \Lambda^{3 / 2}} \frac{\widehat{\chi}_{\Lambda}(k)}{2 \pi \sqrt{|k|}}\left(e^{-i Z q^{2} k \cdot x_{1}}-1\right) \varepsilon_{\lambda}(k) \cdot \nabla_{x_{1}} \\
& -\frac{i Z^{2}}{2 m_{2} \Lambda^{3 / 2}} \frac{\widehat{\chi}_{\Lambda}(k)}{2 \pi \sqrt{|k|}}\left(e^{-i Z q^{2} k \cdot x_{2}}-1\right) \varepsilon_{\lambda}(k) \cdot \nabla_{x_{2}} \\
& -\frac{i Z}{\Lambda^{3 / 2}} \frac{|k|^{1 / 2} \widehat{\chi}_{\Lambda}(k)}{2 \pi} \varepsilon_{\lambda}(k) \cdot \widetilde{r} .
\end{aligned}
$$

Note that we have set $\widetilde{r}:=x_{1}-Z x_{2}$. Moreover, $\widetilde{W}_{2}$ is $W_{2}$ where the terms $e^{ \pm i Z q^{2} k \cdot x_{j}}$ are replaced by $e^{ \pm i Z q^{2} k \cdot x_{j}}-1$ in $\widetilde{G}_{m, n}$, for $m+n=2$. We define the operators $\widetilde{H}_{a t}(\theta), \widetilde{H}_{0}(\theta), \widetilde{W}_{g}(\theta)$ and $\widetilde{G}_{m, n}(\theta)$ by means of the complex scaling operator $\mathcal{U}_{\theta}$ associated with the dilatations (33). Then, in the same way as for $H_{U}^{V}(\theta)$, one can prove:

Proposition 5. Let $\theta_{0}$ and $g$ be sufficiently small. For $\theta$ in $D\left(0, \theta_{0}\right)$, let $\widetilde{H}_{U}^{V}(\theta):=$ $\widetilde{H}_{0}(\theta)+\widetilde{W}_{g}(\theta)$ on $D\left(\widetilde{H}_{0}\right)$. Then $\widetilde{H}_{U}^{V}(\theta)$ fulfills Hypotheses $\left(\mathcal{H}_{a n}\right)$, $\left(\mathcal{H}_{-1 / 2}\right)$ and $\left(\mathcal{H}_{1 / 2}\right)$, with

$$
J_{1 / 2}(k) \leq \frac{\text { Cste }}{g}|k|^{1 / 2} e^{-k^{2} / \Lambda^{2}} .
$$

The factor of order $g^{-1}$ in the bound (51) is due to the last term in (50) which is relatively bounded with respect to $\left|\widetilde{H}_{a t}+i\right|^{1 / 2} / g$. This appears to be a problem, because we require that all the terms of the perturbation $\widetilde{W}_{g}:=g \widetilde{W}_{1}+g^{2} \widetilde{W}_{2}$ are small compared to the unperturbed Hamiltonian $\widetilde{H}_{0}:=\widetilde{H}_{a t}+H_{f}$, when $g$ is small. To avoid this difficulty, in a way similar to what is done in [3], we consider a simplified model where a spatial cutoff is imposed on $\widetilde{W}_{g}$, which restricts the position of the electron to bounded distances from the position of the nucleus. More precisely, we replace $\widetilde{W}_{g}$ by $\widetilde{W}_{g ; \text { reg }}$ where

$$
\widetilde{W}_{g ; \text { reg }}:=\chi_{r_{0}}(r) \widetilde{W}_{g} .
$$

Here $\chi_{r_{0}}$ is a cutoff that, for concreteness, we choose as $\chi_{r_{0}}(r):=e^{-r^{2} / r_{0}^{2}}$, where $r_{0}$ is some arbitrary positive real number. Then we define the Hamiltonian $\widetilde{H}_{U ; \text { reg }}^{V}$ by

$$
\widetilde{H}_{U ; \mathrm{reg}}^{V}:=\widetilde{H}_{0}+\widetilde{W}_{g ; \mathrm{reg}},
$$

and one can verify that:

Proposition 6. Let $\theta_{0}$ and $g$ be sufficiently small. For $\theta$ in $D\left(0, \theta_{0}\right)$, let $\widetilde{H}_{U ; \mathrm{reg}}^{V}(\theta):=$ $\widetilde{H}_{0}(\theta)+\widetilde{W}_{g ; \mathrm{reg}}(\theta)$ on $D\left(\widetilde{H}_{0}\right)$. Then $\widetilde{H}_{U ; \mathrm{reg}}^{V}(\theta)$ fulfills Hypotheses $\left(\mathcal{H}_{a n}\right),\left(\mathcal{H}_{-1 / 2}\right)$ and $\left(\mathcal{H}_{1 / 2}\right)$, with

$$
J_{-1 / 2}(k):=\text { Cste }|k|^{-1 / 2} e^{-k^{2} / \Lambda^{2}}, \quad J_{1 / 2}(k):=\text { Cste }|k|^{1 / 2} e^{-k^{2} / \Lambda^{2}} .
$$




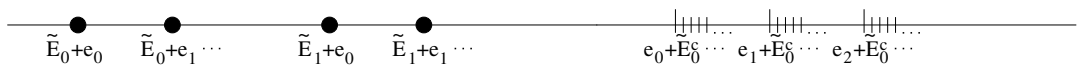

FigURE 4. Spectrum of the new atomic Hamiltonian $\widetilde{H}_{a t}$ for $Z=1$.

Henceforth, we redefine $\widetilde{W}_{g}:=\widetilde{W}_{g ; \text { reg }}$ and $\widetilde{H}_{U}^{V}:=\widetilde{H}_{U ; \text { reg }}^{V}$.

To conclude with this subsection, we describe the spectrum of the "new" atomic Hamiltonian $\widetilde{H}_{a t}$. Assume that we are dealing with the hydrogen atom, that is $Z=1$. Then (48) implies

$$
\begin{aligned}
\widetilde{H}_{a t}= & \frac{p^{2}}{2 \mu}+V+\mathrm{C}^{2} r^{2}+\frac{P^{2}}{2 M}+U \\
& +2 g^{2} \frac{1}{\Lambda^{3}} \int_{\mathbb{R}^{3}} \frac{\widehat{\chi}_{\Lambda}(k)^{2}}{\pi^{2}|k|}\left[\frac{1}{2 m_{1}} \sin ^{2}\left(q^{2} k \cdot x_{1} / 2\right)+\frac{1}{2 m_{2}} \sin ^{2}\left(q^{2} k \cdot x_{2} / 2\right)\right] d k,
\end{aligned}
$$

where $\mathrm{C}$ is positive. One can see that the spectrum of $p^{2} / 2 \mu+V+\mathrm{C} g^{2} r^{2}$ is discrete: the eigenvalues $E_{l}$ of $p^{2} / 2 \mu+V$ are slightly shifted to $\widetilde{E}_{l}$, and the continuous spectrum of $p^{2} / 2 \mu+V$ turns into a non-decreasing sequence of discrete eigenvalues $\widetilde{E}_{l}^{\mathrm{c}}(g), l \geq 1$, such that

$$
\widetilde{E}_{l}^{c}(g)-\widetilde{E}_{l-1}^{c}(g) \underset{g \rightarrow 0}{\rightarrow} 0 .
$$

Thus the spectrum of $p^{2} / 2 \mu+V+\mathrm{Cg}^{2} r^{2}+P^{2} / 2 M+U$ is purely discrete, and the same holds for $\widetilde{H}_{a t}$ : its eigenvalues are $\widetilde{E}_{l}+e_{n}$ and $\widetilde{E}_{l}^{c}+e_{n}$, slightly perturbed by the last term in (55). We still denote by $\widetilde{E}_{l}+e_{n}$ and $\widetilde{E}_{l}^{c}+e_{n}$ the eigenvalues of $\widetilde{H}_{a t}$. Hence we obtain the spectrum of $\widetilde{H}_{a t}$ as described in Figure 4 .

Note that, if $Z>1$, we can not use the same argument to state that the essential spectrum of $p^{2} / 2 \mu+V$ turns into discrete spectrum. However, we still have that the eigenvalues $E_{l}+e_{n}$ of $H_{a t}$ are slightly shifted, remaining eigenvalues of $\widetilde{H}_{a t}$. Since we only study such eigenvalues in the sequel (we shall not study the behavior of $\widetilde{E}_{l}^{c}+e_{n}$ ), the case $Z>1$ can be treated in the same way as the case $Z=1$.

\section{Absolute continuity of the spectrum and existence of resonances}

We proved in the previous section that the Pauli-Fierz Hamiltonian associated with the model of the confined hydrogen atom, $H_{U}^{V}$, fulfills Hypotheses $\left(\mathcal{H}_{a n}\right)$ and $\left(\mathcal{H}_{-1 / 2}\right)$, whereas the regularized Hamiltonian, $\widetilde{H}_{U}^{V}$, fulfills Hypotheses $\left(\mathcal{H}_{a n}\right)$, $\left(\mathcal{H}_{-1 / 2}\right)$ and $\left(\mathcal{H}_{1 / 2}\right)$. From these results, considering operators $H_{g}$ of the form introduced in Subsection 1.2, we shall obtain in this section the absolute continuity of $\sigma\left(H_{U}^{V}\right)$ on an interval, and the existence of resonances for $\widetilde{H}_{U}^{V}$.

\subsection{Preliminaries. Absolute continuity of the spectrum}

Recall from Subsection 1.2 that $H_{g}=H_{0}+W_{g}=H_{a t} \otimes \mathbf{1}+\mathbf{1} \otimes H_{f}+W_{g}$. Consider an eigenvalue $\lambda_{j, 0}$ of $H_{a t}$ strictly located between the ground state energy $\lambda_{0,0}$ and 
the ionization threshold $\Sigma$. We set $\delta_{j}:=\operatorname{dist}\left(\lambda_{j, 0}, \sigma\left(H_{a t}\right) \backslash\left\{\lambda_{j, 0}\right\}\right)$. The projection $P_{a t, j}(\theta)$ onto the eigenspace of $H_{a t}(\theta)$ corresponding to $\lambda_{j, 0}$, and $\bar{P}_{a t, j}(\theta)$, are defined by

$$
P_{a t, j}(\theta):=\frac{i}{2 \pi} \int_{\left|z-\lambda_{j, 0}\right|=\frac{\delta_{j}}{2}} \frac{d z}{H_{a t}(\theta)-z}, \quad \bar{P}_{a t, j}(\theta):=1-P_{a t, j}(\theta) .
$$

As in [5], setting $P_{a t, j}:=P_{a t, j}(0), \bar{P}_{a t, j}:=\bar{P}_{a t, j}(0)$, we define the matrices

$$
\begin{aligned}
Z_{j}^{\text {od }} & :=\int_{\underline{\mathbb{R}}^{3}} P_{a t, j} G_{0,1}(\underline{k}) \bar{P}_{a t, j}\left[H_{a t}-\lambda_{j, 0}+|k|-i 0\right]^{-1} \bar{P}_{a t, j} G_{1,0}(\underline{k}) P_{a t, j} d \underline{k}, \\
Z_{j}^{d} & :=\int_{\underline{\mathbb{R}}^{3}} P_{a t, j} G_{0,1}(\underline{k}) P_{a t, j} G_{1,0}(\underline{k}) P_{a t, j} \frac{d \underline{k}}{|k|} .
\end{aligned}
$$

For $\theta \in D\left(0, \theta_{0}\right)$, we set

$$
Z_{j}^{\text {od }}(\theta):=\mathcal{U}_{\theta} Z_{j}^{\text {od }} \mathcal{U}_{\theta}^{-1}, \quad Z_{j}^{\mathrm{d}}(\theta):=\mathcal{U}_{\theta} Z_{j}^{\mathrm{d}} \mathcal{U}_{\theta}^{-1}, \quad Z_{j}(\theta):=Z_{j}^{\mathrm{d}}(\theta)-Z_{j}^{\text {od }}(\theta) .
$$

Let $\Gamma_{j}:=\min \left\{\sigma\left(\operatorname{Im}\left(Z_{j}^{\text {od }}\right)\right)\right\}$; then for the needs of the proof, we have to require the following hypothesis, related to the Fermi golden rule:

$$
\left(\mathcal{H}_{\Gamma_{j}}\right) \quad \Gamma_{j}>0 \text {. }
$$

We shall verify in the appendix that Hypothesis $\left(\mathcal{H}_{\Gamma_{1}}\right)$ is indeed satisfied in the case of $H_{U}^{V}$, for suitably chosen parameters. Now, to simplify, we assume moreover that:

$$
\left(\mathcal{H}_{\Gamma_{j}}^{\prime}\right) \quad \Gamma_{j}>0 \text { and } \Gamma_{j} \text { is a simple eigenvalue of } \operatorname{Im}\left(Z_{j}^{\text {od }}\right) .
$$

Let $\phi_{j, 0}$ be a normalized eigenvector associated with the eigenvalue $\Gamma_{j}$ of $\operatorname{Im}\left(Z_{j}^{\text {od }}\right)$; we define

$$
\Delta_{j}:=\operatorname{Re}\left[\left(\phi_{j, 0}, Z_{j} \phi_{j, 0}\right)\right] .
$$

Let $\mathcal{S}_{j}, \mathcal{R}_{j}(\varepsilon, C)$ denote the following sets:

$$
\begin{aligned}
\mathcal{S}_{j} & :=\lambda_{j, 0}+g^{2}\left(\Delta_{j}-i \Gamma_{j}\right)-i \mathcal{Q}_{j}, \\
\mathcal{R}_{j}(\varepsilon, C) & :=\mathcal{S}_{j}+e^{-\theta} \mathbb{R}_{+}+D\left(0, C g^{2+\varepsilon}\right),
\end{aligned}
$$

where $\varepsilon$ is a small positive constant and $C \in \mathbb{R}_{+}$. The set $\mathcal{Q}_{j}:=\{z \in \mathbb{C} \mid-\mu \leq$ $\arg (z) \leq \mu\}$ (for some $0<\mu<\pi / 2$ ) is supposed (by Hypothesis $\left(\mathcal{H}_{\Gamma_{j}}^{\prime}\right)$ ) to be such that

$$
\left\{\left(\phi, Z_{j} \phi\right),\|\phi\|=1\right\} \subset \Delta_{j}-i \Gamma_{j}-i \mathcal{Q}_{j} .
$$

Finally, the set $\mathcal{A}_{j}(\varepsilon)$ is defined by

$$
A_{j}(\varepsilon):=\left\{z \in \mathbb{C}, \operatorname{Re}(z) \in \mathcal{I}_{j},|\operatorname{Im}(z)| \leq \frac{1}{2} g^{2-2 \varepsilon} \sin \nu\right\},
$$

where $\mathcal{I}_{j}$ is the interval $] \lambda_{j, 0}-\delta_{j} / 2 ; \lambda_{j, 0}+\delta_{j} / 2[$. The following result is proved as in [5] (see also Proposition 11 below for the proof of the existence of a Feshbach operator associated with $\left.H_{g}(\theta)\right)$ : 


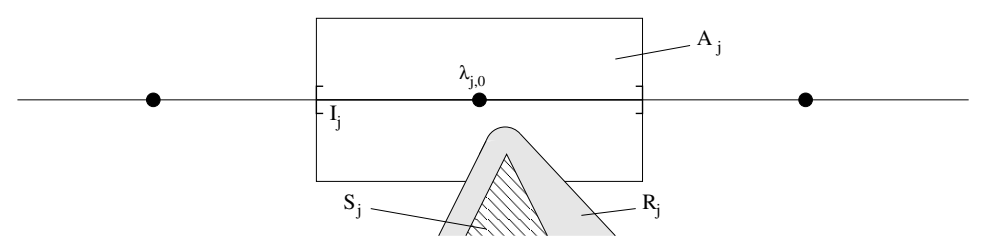

FiguRE 5. The resolvent set of $H_{g}(\theta): \mathcal{A}_{j} \backslash \mathcal{R}_{j} \subset \rho\left(H_{g}(\theta)\right)$ (see Theorem 7).

Theorem 7. Let $g>0$ sufficiently small. Assume that Hypotheses $\left(\mathcal{H}_{\text {an }}\right)$ and $\left(\mathcal{H}_{-1 / 2}\right)$ hold for a sufficiently small $\theta_{0}$. Let $0<\varepsilon<1 / 3$ and fix $\theta=i \nu$ in $D\left(0, \theta_{0}\right)$ with $\nu>0$. Assume that $\left(\mathcal{H}_{\Gamma_{j}}^{\prime}\right)$ is fulfilled. Then there exists a positive constant $C$ such that

$$
\mathcal{A}_{j}(\varepsilon) \backslash \mathcal{R}_{j}(\varepsilon, C) \subset \rho\left(H_{g}(\theta)\right)
$$

where $\rho\left(H_{U}^{V}(\theta)\right)$ denotes the resolvent set of $H_{g}(\theta)$ (see Figure 5). This implies that the spectrum of $H_{g}$ is absolutely continuous in $\mathcal{I}_{j}$.

As a corollary of Theorem 7, we obtain Theorem 2, which implies by Proposition 4:

Corollary 8. Let $g>0$ sufficiently small. Let $E_{U}^{V}$ be the ground state energy of $H_{U}^{V}$. Assume that Hypotheses $\left(\mathcal{H}_{\Gamma_{j}}\right)$ hold for all non-perturbed eigenvalues $\lambda_{j, 0}$ located below $e_{0}$. Then $\sigma\left(H_{U}^{V}\right)$ is absolutely continuous on $\left[E_{U}^{V}, e_{0}\right] \backslash \mathcal{V}$, where $\mathcal{V}$ is a neighborhood of order $O\left(g^{2}\right)$ of $\left\{E_{U}^{V}\right\} \cup\left\{e_{0}\right\}$.

\subsection{The smooth Feshbach map applied to $H_{g}(\theta)$}

Henceforth, to simplify, we suppose that the eigenvalue $\lambda_{j, 0}$ of $H_{a t}$ is non-degenerate and located at 0 . The complex parameter $\theta$ is fixed to $\theta:=i \nu$ for some $\nu>0$. For any $\rho_{0}>0$ such that $\rho_{0} \leq\left(\delta_{j} \sin \nu\right) / 2<1$, we define the functions of $H_{f}$, $\chi_{\rho_{0}}\left(H_{f}\right)$ and $\bar{\chi}_{\rho_{0}}\left(H_{f}\right)$, by

$$
\begin{aligned}
\chi_{\rho_{0}}\left(H_{f}\right) & :=\sin \left[\frac{\pi}{2} \Theta\left(H_{f} / \rho_{0}\right)\right], \\
\bar{\chi}_{\rho_{0}}\left(H_{f}\right) & :=\sqrt{1-\chi_{\rho_{0}}^{2}\left(H_{f}\right)}=\cos \left[\frac{\pi}{2} \Theta\left(H_{f} / \rho_{0}\right)\right],
\end{aligned}
$$

where $\Theta \in \mathrm{C}_{0}^{\infty}([0, \infty[;[0,1])$ is such that $\Theta=1$ on $[0,3 / 4[$ and $\Theta=0$ on $[1, \infty[$. Next we use (56) and (65) to define

$$
P(\theta):=P_{a t, j}(\theta) \otimes \chi_{\rho_{0}}\left(H_{f}\right), \quad \bar{P}(\theta):=P_{a t, j}(\theta) \otimes \bar{\chi}_{\rho_{0}}\left(H_{f}\right)+\bar{P}_{a t, j}(\theta) \otimes \mathbf{1} .
$$

Note that (66) implies $P(\theta)^{2}+\bar{P}(\theta)^{2}=\mathbf{1}$. A key point in the proof of Theorem 7 is to get the existence of the Feshbach operator (or smooth Feshbach operator [2]) 
$\mathcal{F}_{P(\theta)}\left(H_{g}(\theta)-z, H_{0}(\theta)-z\right)$ defined by

$$
\begin{aligned}
\mathcal{F}_{P(\theta)}\left(H_{g}(\theta)-z, H_{0}(\theta)-z\right):= & \left(H_{0}(\theta)-z\right)+P(\theta) W_{g}(\theta) P(\theta) \\
& -P(\theta) W_{g}(\theta) \bar{P}(\theta)\left[\left(H_{0}(\theta)-z\right)\right. \\
& \left.+\bar{P}(\theta) W_{g}(\theta) \bar{P}(\theta)\right]^{-1} \bar{P}(\theta) W_{g}(\theta) P(\theta) .
\end{aligned}
$$

where $z$ is a spectral parameter belonging to $D_{\rho_{0} / 2}:=\left\{z \in \mathbb{C},|z| \leq \rho_{0} / 2\right\}$. Here we give two lemmas that lead to the existence of $\mathcal{F}_{P(\theta)}\left(H_{g}(\theta)-z, H_{0}(\theta)-z\right)$.

Lemma 9. Assume that Hypothesis $\left(\mathcal{H}_{-1 / 2}\right)$ holds for some $\theta_{0}>0$ sufficiently small. Then there exists a positive constant $\mathrm{C}$ such that for all $\theta \in D\left(0, \theta_{0}\right), \rho>0$, and $m, m^{\prime}, n, n^{\prime} \in \mathbb{N}, m+n \in\{1,2\}, m+m^{\prime}+n+n^{\prime}=2$ :

$$
\left\|\left|H_{a t}+i\right|^{-\frac{m^{\prime}}{2}}\left[H_{f}+\rho\right]^{-\frac{m}{2}} W_{m, n}(\theta)\left[H_{f}+\rho\right]^{-\frac{n}{2}}\left|H_{a t}+i\right|^{-\frac{n^{\prime}}{2}}\right\| \leq \mathrm{C} .
$$

Proof. (68) follows from Hypothesis $\left(\mathcal{H}_{-1 / 2}\right)$ and well-known "Pull-through formulas"; we refer for instance to [5] for details. See also Lemma 14 below.

Lemma 10. Assume that Hypothesis $\left(\mathcal{H}_{\text {an }}\right)$ holds for some $\theta_{0}>0$ sufficiently small. Then there exists a positive constant $\mathrm{C}$ such that for all $\theta=i \nu \in D\left(0, \theta_{0}\right)$, $0<\rho_{0}<\left(\delta_{j} \sin \nu\right) / 2, z \in D_{\rho_{0} / 2}$, and $m, m^{\prime}, n, n^{\prime} \in \mathbb{N}, m+m^{\prime}+n+n^{\prime}=2$ :

$$
\begin{aligned}
\|\left|H_{a t}+i\right|^{\frac{m^{\prime}}{2}}\left[H_{f}+\rho_{0}\right]^{\frac{m}{2}} \bar{P}(\theta)\left[\left(H_{0}(\theta)-z\right) \mathbf{1}_{\operatorname{Ran}(\bar{P}(\theta))}\right]^{-1} & \\
\bar{P}(\theta)\left[H_{f}+\rho_{0}\right]^{\frac{n}{2}}\left|H_{a t}+i\right|^{\frac{n^{\prime}}{2}} \| & \leq \frac{\mathrm{C}}{\delta_{j} \sin \nu} \rho_{0}^{-\frac{m^{\prime}+n^{\prime}}{2}} .
\end{aligned}
$$

Proof. Let us prove (69) for $m=n^{\prime}=1$. The other cases could be obtained similarly. First consider the contribution of $\bar{P}_{a t, j}(\theta) \otimes \mathbf{1}$ in $\bar{P}(\theta)$. We write $\bar{P}_{a t, j}(\theta)$ as

$$
\bar{P}_{a t, j}(\theta)=\sum_{l=0}^{j-1} P_{a t, l}(\theta)+P_{a t,>j}(\theta),
$$

where $P_{a t,>j}(\theta):=1-\sum_{i=0}^{j} P_{a t, l}(\theta)$. Hypothesis $\left(\mathcal{H}_{a n}\right)$ implies that $\left[H_{a t}+i\right] P_{a t, l}(\theta)$ is bounded for any $l \leq j$. Hence by the Spectral Theorem,

$$
\begin{array}{r}
\sum_{l=0}^{j-1}\left\|\left|H_{a t}+i\right|^{1 / 2} P_{a t, l}(\theta) \otimes \mathbf{1}\left[\left(H_{0}(\theta)-z\right) \mathbf{1}_{\operatorname{Ran}(\bar{P}(\theta))}\right]^{-1}\left[H_{f}+\rho_{0}\right]^{1 / 2}\right\| \\
\leq \frac{\mathrm{C}}{\delta_{j} \sin \nu} .
\end{array}
$$

To estimate the contribution of $P_{a t,>j}(\theta)$, note that

$$
\begin{aligned}
\left(P_{a t,>j}(\theta) \otimes \mathbf{1}\right)\left[H_{0}(\theta)-z\right]= & \left(P_{a t,>j} \otimes \mathbf{1}\right)\left[H_{a t}+e^{-\theta} H_{f}-z\right] \\
& +\left(P_{a t,>j}(\theta) \otimes \mathbf{1}\right)\left[H_{a t}(\theta)-H_{a t}\right] \\
& +\left(\left(P_{a t,>j}(\theta)-P_{a t,>j}\right) \otimes \mathbf{1}\right)\left[H_{a t}+e^{-\theta} H_{f}-z\right],
\end{aligned}
$$


where $P_{a t,>j}:=P_{a t,>j}(0)$. Since $\left\|\left[\left(P_{a t,>j} \otimes \mathbf{1}\right)\left[H_{a t}+e^{-\theta} H_{f}-z\right]\right]^{-1}\right\| \leq$ Cste $\delta_{j}^{-1}$, a Neumann series expansion, Hypothesis $\left(\mathcal{H}_{a n}\right)$ and the Spectral Theorem yield

$$
\left\|\left|H_{a t}+i\right|^{1 / 2} P_{a t,>j}(\theta) \otimes \mathbf{1}\left[\left(H_{0}(\theta)-z\right) \mathbf{1}_{\operatorname{Ran}(\bar{P}(\theta))}\right]^{-1}\left[H_{f}+\rho_{0}\right]^{1 / 2}\right\| \leq \frac{\mathrm{C}}{\delta_{j}} .
$$

Finally, using that $\left[H_{a t}+i\right] P_{a t, j}(\theta)$ is bounded together with the Spectral Theorem, the contribution of $P_{a t, j}(\theta) \otimes \bar{\chi}_{\rho_{0}}\left(H_{f}\right)$ is estimated as

$$
\begin{aligned}
\left\|\left|H_{a t}+i\right|^{1 / 2} P_{a t, j}(\theta) \otimes \bar{\chi}_{\rho_{0}}\left(H_{f}\right)\left[\left(H_{0}(\theta)-z\right) \mathbf{1}_{\operatorname{Ran}(\bar{P}(\theta))}\right]^{-1}\left[H_{f}+\rho_{0}\right]^{1 / 2}\right\| \\
\leq \frac{\mathrm{C}}{\delta_{j} \sin \nu} \rho_{0}^{-1 / 2} .
\end{aligned}
$$

Hence the proof is complete if $m=n^{\prime}=1$. The other cases are similar.

From Lemmas 9 and 10, we obtain the existence and isospectrality of the Feshbach operator (67) in the following sense:

Proposition 11. Let $g>0$ sufficiently small. Assume that Hypotheses $\left(\mathcal{H}_{a n}\right)$ and $\left(\mathcal{H}_{-1 / 2}\right)$ hold for some $\theta_{0}>0$ sufficiently small. Let $0<\rho_{0}<\left(\delta_{j} \sin \nu\right) / 2$. Then, for all $z \in D_{\rho_{0} / 2}$, the Feshbach operator (67) is well-defined. Moreover, $z$ is an eigenvalue of $H_{g}(\theta)$ with multiplicity $m$ if and only if 0 is an eigenvalue of $\left.\mathcal{F}_{P(\theta)}\left(H_{g}(\theta)-z, H_{0}(\theta)-z\right)\right|_{\operatorname{Ran}(P(\theta))}$ with the same multiplicity.

Proof. Expanding the resolvent in the rhs of (67) into a Neumann series yields

$$
\begin{aligned}
& {\left[\left(H_{0}(\theta)-z\right) \mathbf{1}_{\operatorname{Ran}(\bar{P}(\theta))}+\bar{P}(\theta) W_{g}(\theta) \bar{P}(\theta)\right]^{-1}} \\
& =\left[\left(H_{0}(\theta)-z\right) \mathbf{1}_{\operatorname{Ran}(\bar{P}(\theta))}\right]^{-1} \sum_{n \geq 0}\left(-\bar{P}(\theta) W_{g}(\theta) \bar{P}(\theta)\left[\left(H_{0}(\theta)-z\right) \mathbf{1}_{\operatorname{Ran}(\bar{P}(\theta))}\right]^{-1}\right)^{n} .
\end{aligned}
$$

The rhs of the last equation is well-defined for $g \rho_{0}^{-1 / 2}$ sufficiently small, which can be seen by using the estimates of Lemmas 9 and 10 . One can prove similarly that $\mathcal{F}_{P(\theta)}\left(H_{g}(\theta)-z, H_{0}(\theta)-z\right)$ is well-defined and satisfies all the assumptions of [11]. The isospectrality then follows by [11].

Using (75), our aim in the next subsection will be to prove that the Feshbach operator (67) can be identified with an element of a suitably chosen Banach space constructed in a way similar to the one in [2]. The main property of this Banach space that we shall require is that the operator coming from the interaction is irrelevant under renormalization. 


\subsection{A Banach space of Hamiltonians}

For the convenience of the reader, we describe precisely the Banach space of Hamiltonians constructed here in a way similar to [2]. We set:

$$
\mathcal{W}_{\geq 0}^{\#}:=\mathbb{C} \oplus \mathcal{T} \oplus \mathcal{W}_{\geq 1}^{\#}:=\mathbb{C} \oplus \mathcal{T} \oplus \bigoplus_{M+N \geq 1} \mathcal{W}_{M, N}^{\#},
$$

where

$$
\mathcal{T}:=\left\{f \in \mathrm{C}^{1}([0,1]), f(0)=0,\|f\|_{\mathcal{T}}:=\sup _{\gamma \in[0,1]}\left|f^{\prime}(\gamma)\right|<\infty\right\}
$$

and

$$
\begin{aligned}
\mathcal{W}_{M, N}^{\#}:=\{ & f_{M, N}:[0,1] \times \underline{B}_{1}^{M} \times \underline{B}_{1}^{N} \rightarrow \mathbb{C} \text { such that: } \\
& * f_{M, N}\left(\cdot ; \underline{k}^{(M)} ; \underline{\tilde{k}}^{(N)}\right) \in \mathrm{C}^{1}([0,1]) \text { for every }\left(\underline{k}^{(M)} ; \underline{\tilde{k}}^{(N)}\right) \in \underline{B}_{1}^{M+N}, \\
& * f_{M, N}\left(\gamma ; \underline{k}^{(M)} ; \underline{\tilde{k}}^{(N)}\right) \text { is symmetric w.r.t. } \underline{k}^{(M)} \text { and } \underline{\tilde{k}}^{(N)}, \\
& \left.*\left\|f_{M, N}\right\|^{\#}:=\left\|f_{M, N}\right\|+\left\|\partial_{\gamma} f_{M, N}\right\|<\infty\right\} .
\end{aligned}
$$

Here $\underline{B}_{1}=B_{1} \times\{1,2\}$ where $B_{1}$ is the unit ball in $\mathbb{R}^{3}$. Moreover $\partial_{\gamma} f_{M, N}$ is the partial derivative of $f_{M, N}$ with respect to the first variable, and

$$
\left\|f_{M, N}\right\|:=\sup _{[0,1] \times \underline{B}_{1}^{M+N}}\left|f_{M, N}\left(\gamma ; \underline{k}^{(M)} ; \underline{\tilde{k}}^{(N)}\right)\right| \prod_{i=1}^{M}\left|k_{i}\right|^{-1 / 2} \prod_{j=1}^{N}\left|\tilde{k}_{j}\right|^{-1 / 2} .
$$

Note that we have used the notations

$$
\underline{k}^{(M)}:=\left(\underline{k}_{1}, \ldots, \underline{k}_{M}\right) \in \underline{\mathbb{R}}^{3 M}, \quad \underline{\tilde{k}}^{(N)}:=\left(\underline{\tilde{k}}_{1}, \ldots, \underline{\tilde{k}}_{N}\right) \in \underline{\mathbb{R}}^{3 N} .
$$

Note also that, in [2], a $\mathrm{L}^{2}$-norm is used instead of the $\mathrm{L}^{\infty}$-norm considered in (79). Next, the space $\mathcal{W}_{\geq 1}^{\#}:=\left\{\underline{w}:=\left(w_{M, N}\right)_{M+N \geq 1}\right\}$ is equipped with the norm

$$
\|\underline{w}\|_{\zeta, 1}^{\#}:=\sum_{M+N \geq 1} \zeta^{-(M+N)}\left\|w_{M, N}\right\|^{\#},
$$

where $0<\zeta<1$ is a parameter that we will precise below. Defining

$$
\mathcal{W}_{0,0}^{\#}:=\left\{f_{0,0} \in \mathrm{C}^{1}([0,1]),\left\|f_{0,0}\right\|^{\#}:=\left\|f_{0,0}\right\|_{\infty}+\left\|\partial_{\gamma} f_{0,0}\right\|_{\infty}<\infty\right\},
$$

one can see that there is a natural isomorphism between the Banach spaces $\mathbb{C} \oplus \mathcal{T}$ and $\mathcal{W}_{0,0}^{\#}$, so that we identify $\mathbb{C} \oplus \mathcal{T}$ and $\mathcal{W}_{0,0}^{\#}$. Thus, we can write an element of $\mathcal{W}_{\geq 0}^{\#}$ as $\underline{w}:=\left(w_{M, N}\right)_{M+N \geq 0}$, and $\mathcal{W}_{\geq 0}^{\#}$ is equipped with the norm

$$
\|\underline{w}\|_{\zeta}^{\#}:=\sum_{M+N \geq 0} \zeta^{-(M+N)}\left\|w_{M, N}\right\|^{\#} .
$$

Now, we want to identify an element of $\mathcal{W}_{\geq 0}^{\#}$ with an operator on the Hilbert space

$$
\mathcal{H}_{\text {red }}:=\mathbf{1}_{H_{f}<1} \mathcal{F}_{s} \text {. }
$$


To this end, we define for $\underline{w} \in \mathcal{W}_{\geq 0}^{\#}$ :

$$
H(\underline{w}):=\sum_{M+N \geq 0} W_{M, N}(\underline{w}):=w_{0,0}\left(H_{f}\right) \mathbf{1}_{H_{f}<1}+\sum_{M+N \geq 1} W_{M, N}(\underline{w}),
$$

where for $M+N \geq 1$ :

$$
\begin{aligned}
& W_{M, N}(\underline{w}):=\mathbf{1}_{H_{f}<1} \\
& \quad \times \int_{\underline{B}_{1}^{M+N}} a^{*}\left(\underline{k}^{(M)}\right) w_{M, N}\left[H_{f} ; \underline{k}^{(M)} ; \underline{\tilde{k}}^{(N)}\right] a\left(\underline{\tilde{k}}^{(N)}\right) d \underline{k}^{(M)} d \underline{\tilde{k}}^{(N)} \mathbf{1}_{H_{f}<1} .
\end{aligned}
$$

Note that in (86), we have used the notations

$$
\begin{aligned}
a^{*}\left(\underline{k}^{(M)}\right) & :=\prod_{j=1}^{M} a^{*}\left(\underline{k}_{j}\right), & a\left(\underline{\tilde{k}}^{(N)}\right) & :=\prod_{j=1}^{N} a\left(\underline{\tilde{k}}_{j}\right), \\
d \underline{k}^{(M)} & :=d \underline{k}_{1} \ldots d \underline{k}_{M}, \quad & d \underline{\tilde{k}}^{(N)} & :=d \underline{\tilde{k}}_{1} \ldots d \underline{\tilde{k}}_{N} .
\end{aligned}
$$

The following proposition can be proved as in [2]; it shows that any operator of $\mathcal{H}_{\text {red }}$ written as in (85) can be identified with an element of the Banach space $\mathcal{W}_{\geq 0}^{\#}$.

Proposition 12. Pick $\zeta$ such that $0<\zeta<1$. Then the map $H$ defined in (85) is an injective embedding from $\mathcal{W}_{\geq 0}^{\#}$ into $\mathcal{B}\left[\mathcal{H}_{\text {red }}\right]$, the set of bounded operators on $\mathcal{H}_{\text {red }}$. Moreover, we have

$$
\|H(\underline{w})\|_{\mathcal{H}_{\text {red }}} \leq\|\underline{w}\|_{\zeta}^{\#} .
$$

To control the dependence of the operators that we shall study on the spectral parameter $z$, we introduce the Banach space

$$
\mathcal{W}_{\geq 0}:=\left\{\underline{w}[\cdot]: D_{1 / 2} \rightarrow \mathcal{W}_{\geq 0}^{\#}, \underline{w}[\cdot] \text { is analytic }\right\},
$$

where $D_{1 / 2}$ denotes the disc $\{z \in \mathbb{C},|z| \leq 1 / 2\}$. It is equipped with the norm

$$
\|\underline{w}[\cdot]\|_{\zeta}:=\sup _{z \in D_{1 / 2}}\|\underline{w}[z]\|_{\zeta}^{\#} .
$$

Likewise, $H\left(\mathcal{W}_{\geq 0}\right)$ denotes the Banach space

$$
H\left(\mathcal{W}_{\geq 0}\right):=\left\{H(\underline{w}[\cdot]): D_{1 / 2} \rightarrow H\left(\mathcal{W}_{\geq 0}^{\#}\right), H(\underline{w}[\cdot]) \text { is analytic }\right\},
$$

with the norm

$$
\|H(\underline{w}[\cdot])\|:=\sup _{z \in D_{1 / 2}}\|H(\underline{w}[z])\|_{\mathcal{H}_{\mathrm{red}}} .
$$

This is on this Banach space (with a slight difference in the choice of the norm (79) as mentioned above) that the renormalization map constructed in [2] acts. 


\subsection{Renormalization and existence of resonances}

Our first aim is to define an operator $H_{(0)}[z]$ which is isospectral to $H_{g}(\theta)$, and which belongs to $H\left(\mathcal{W}_{\geq 0}\right)$. In the same way as in [3], we define, for any $\xi$ in $D_{\rho_{0} / 2}$,

$$
\begin{aligned}
\widetilde{H}_{\mathrm{eff}}[\xi]: & =e^{i \nu} \mathbf{1}_{H_{f}<\rho_{0}}\left\langle\mathcal{F}_{P(\theta)}\left(H_{g}(\theta)-\xi, H_{0}(\theta)-\xi\right)+\xi\right\rangle_{a t} \mathbf{1}_{H_{f}<\rho_{0}} \\
& =H_{f} \mathbf{1}_{H_{f}<\rho_{0}}+e^{i \nu}\langle\ldots\rangle_{a t},
\end{aligned}
$$

where $\langle\ldots\rangle_{a t}$ denotes

$$
\begin{aligned}
\left\langleP ( \theta ) \left[ W_{g}(\theta)-W_{g}(\theta) \bar{P}(\theta)\left[\left(H_{0}(\theta)-\xi\right)+\bar{P}(\theta) W_{g}(\theta) \bar{P}(\theta)\right]^{-1}\right.\right. & \left.\left.\bar{P}(\theta) W_{g}(\theta)\right] P(\theta)\right\rangle_{a t},
\end{aligned}
$$

and where, for a bounded operator $A$ on $\mathrm{L}^{2}\left(\mathbb{R}^{6}\right) \otimes \mathcal{F}_{s}$, the operator $\langle A\rangle_{a t}$ on $\mathcal{F}_{s}$ is defined as the operator associated with the bounded quadratic form

$$
q_{\langle A\rangle_{a t}}(\Phi, \Psi):=\left(\phi_{0}(\theta) \otimes \Phi, A \phi_{0}(\theta) \otimes \Psi\right) .
$$

Here, $\phi_{0}(\theta)$ denotes a normalized eigenstate associated with the non-degenerate eigenvalue 0 of $H_{a t}(\theta)$. Observe that $\widetilde{H}_{\text {eff }}[\xi]$ defines an operator on $\mathbf{1}_{H_{f}<\rho_{0}} \mathcal{F}_{s}$. To obtain an operator on $\mathcal{H}_{\text {red }}$, we scale the photons momenta through a unitary transformation $\mathcal{U}_{\rho_{0}}$ such that $k \mapsto \rho_{0} k$. Then we set

$$
H_{\text {eff }}[\xi]:=\frac{1}{\rho_{0}} \mathcal{U}_{\rho_{0}} \widetilde{H}_{\mathrm{eff}}[\xi] \mathcal{U}_{\rho_{0}}^{*}=H_{f} \mathbf{1}_{H_{f}<1}+\frac{e^{i \nu}}{\rho_{0}} \mathcal{U}_{\rho_{0}}\langle\ldots\rangle \text { at } \mathcal{U}_{\rho_{0}}^{*},
$$

for all $\xi \in D_{\rho_{0} / 2}$. Finally, to obtain an analytic family of operators $H_{(0)}[z]$ with $z \in D_{1 / 2}$, we map the spectral parameter through the transformation

$$
Z_{(0)}: D_{\rho_{0} / 2} \rightarrow D_{1 / 2}, \quad \xi \mapsto \frac{e^{i \nu}}{\rho_{0}} \xi
$$

Thus, we get a family $H_{(0)}[z]$ which is well-defined as a family of bounded operators on $\mathcal{H}_{\text {red }}$ for all $z \in D_{1 / 2}$ :

$$
\begin{aligned}
& H_{(0)}[z]:=H_{\mathrm{eff}}\left[Z_{(0)}^{-1}(z)\right] \\
& =\frac{e^{i \nu}}{\rho_{0}} \mathbf{1}_{H_{f}<1} \mathcal{U}_{\rho_{0}}\left\langle\mathcal{F}_{P(\theta)}\left(H_{g}(\theta)-Z_{(0)}^{-1}(z), H_{0}(\theta)-Z_{(0)}^{-1}(z)\right)\right\rangle_{a t} \mathcal{U}_{\rho_{0}}^{*} \mathbf{1}_{H_{f}<1} \\
& +z \mathbf{1}_{H_{f}<1}
\end{aligned}
$$

We come now to the main theorem of this section, which, with the help of the results obtained in $[3,4]$ and [2], will lead to the existence of resonances:

Theorem 13. Let $g>0$ sufficiently small. Assume that Hypotheses $\left(\mathcal{H}_{a n}\right),\left(\mathcal{H}_{-1 / 2}\right)$ and $\left(\mathcal{H}_{1 / 2}\right)$ hold for some $\theta_{0}>0$ sufficiently small. Let $0<\rho_{0}<\left(\delta_{j} \sin \nu\right) / 2$. 
Choose $\beta, \varepsilon>0$. Then, $H_{(0)}[\cdot]$ belongs to $H\left(\mathcal{W}_{\geq 0}\right)$; furthermore, defining $H_{(0)}[z]:=$ $H\left(\underline{w}_{(0)}[z]\right)$, we have $\underline{w}_{(0)}[\cdot] \in B(\beta, \varepsilon)$, where

$$
\begin{gathered}
B(\beta, \varepsilon):=\left\{\underline{w}[\cdot]=\left(E[\cdot], T[\cdot],\left(w_{M, N}[\cdot]\right)_{M+N \geq 1}\right) \in \mathcal{W}_{\geq 0},\right. \\
\sup _{z \in D_{1 / 2}}\|T[z, \gamma]-\gamma\|_{\mathcal{T}} \leq \beta, \sup _{z \in D_{1 / 2}}|E[z]| \leq \varepsilon \\
\left.\sup _{z \in D_{1 / 2}}\left\|\left(w_{M, N}[z]\right)_{M+N \geq 1}\right\|_{\zeta}^{\#} \leq \varepsilon\right\} .
\end{gathered}
$$

Note that the parameter $\zeta<1$ appearing in the definition of $\mathcal{W}_{\geq 0}$ is chosen such that $\zeta \geq \rho_{0}^{1 / 2}$.

Proof. We sketch the proof and emphasize the main points which differ from $[3,4]$. In particular, Hypothesis $\left(\mathcal{H}_{1 / 2}\right)$ shall be essential here. We begin with considering the operator $\widetilde{H}_{\text {eff }}[\xi]$, defined in (93) for $\xi \in D_{\rho_{0} / 2}$, that we write as

$$
\widetilde{H}_{\mathrm{eff}}[\xi]=\mathbf{1}_{H_{f}<\rho_{0}}\left[\widetilde{E}_{\mathrm{eff}}[\xi]+\widetilde{T}_{\mathrm{eff}}\left[\xi ; H_{f}\right]+\widetilde{W}_{\mathrm{eff}}\left[\xi ; H_{f}\right]\right] \mathbf{1}_{H_{f}<\rho_{0}},
$$

where

$$
\begin{aligned}
\widetilde{E}_{\mathrm{eff}}[\xi] & :=\widetilde{w}_{0,0}^{\mathrm{eff}}[\xi, 0], \\
\widetilde{T}_{\mathrm{eff}}\left[\xi ; H_{f}\right] & :=H_{f}+\widetilde{w}_{0,0}^{\mathrm{eff}}\left[\xi ; H_{f}\right]-\widetilde{w}_{0,0}^{\mathrm{eff}}[\xi, 0], \\
\widetilde{W}_{\mathrm{eff}}\left[\xi ; H_{f}\right] & :=\sum_{M+N \geq 1} \widetilde{W}_{M, N}^{\mathrm{eff}}\left[\xi ; H_{f}\right],
\end{aligned}
$$

and

$$
\left.\widetilde{W}_{M, N}^{\mathrm{eff}}\left[\xi ; H_{f}\right]:=\int_{\underline{\mathbb{R}}^{3(M+N)}} a^{*}\left(\underline{k}^{(M)}\right)\right) \widetilde{w}_{M, N}^{\mathrm{eff}}\left[\xi ; H_{f} ; \underline{k}^{(M)} ; \underline{\tilde{k}}^{(N)}\right] a\left(\underline{\tilde{k}}^{(N)}\right) d \underline{k}^{(M)} d \underline{\tilde{k}}^{(N)} .
$$

Then, assuming that $g$ and $\rho_{0}$ are chosen as in Proposition 11, the Pull-through formula, Wick's theorem and (75) yield (see [4])

$$
\begin{gathered}
\widetilde{w}_{M, N}^{\mathrm{eff}}\left[\xi ; \gamma ; \underline{k}^{(M)} ; \underline{\tilde{k}}^{(N)}\right] \\
=e^{i \nu} \sum_{L=1}^{\infty}(-1)^{L-1} \sum_{\substack{m_{l}+n_{l}+p_{l}+q_{l}=1,2 \\
l=1, \ldots, L}} \delta_{M, \sum_{l=1}^{M} m_{l}} \delta_{N, \sum_{l=1}^{N} n_{l}} \prod_{l=1}^{L}\left(\begin{array}{c}
m_{l}+p_{l} \\
p_{l}
\end{array}\right) \\
\left(\begin{array}{c}
n_{l}+q_{l} \\
q_{l}
\end{array}\right)\left\{\widetilde { D } _ { L } \left[\xi ; H_{f} ;\left\{W_{p_{l}, q_{l}}^{m_{l}, n_{l}} ; \underline{k}_{l}^{\left(m_{l}\right)} ; \underline{\tilde{k}}_{l}^{\left(n_{l}\right)}\right\}_{l=1}^{L} ;\right.\right. \\
\left.\left.\left\{R_{0}^{\bar{P}}\left[H_{f} ; \theta\right]\right\}_{l=1}^{L-1}\right]\right\}_{M, N}^{\mathrm{symm}},
\end{gathered}
$$


where $\left\{f\left[\underline{k}^{(m)} ; \underline{\tilde{k}}^{(n)}\right]\right\}_{m, n}^{\text {symm }}$ denotes the symmetrization of $f$ w.r.t. the variables $\underline{k}^{(m)}$ and $\underline{\tilde{k}}^{(n)}$ :

$$
\left\{f\left[\underline{k}^{(m)}, \underline{\tilde{k}}^{(n)}\right]\right\}_{m, n}^{\mathrm{symm}}:=\frac{1}{m ! n !} \sum_{\pi \in S_{m}} \sum_{\tilde{\pi} \in S_{n}} f\left[\underline{k}_{\pi(1)}, \ldots, \underline{k}_{\pi(m)} ; \underline{\tilde{k}}_{\tilde{\pi}(1)}, \ldots, \underline{\tilde{k}}_{\tilde{\pi}(n)}\right],
$$

and

$$
\begin{gathered}
\widetilde{D}_{L}\left[\xi ; \gamma ;\left\{W_{p_{l}, q_{l}}^{m_{l}, n_{l}} ; \underline{k}_{l}^{\left(m_{l}\right)} ; \underline{\tilde{k}}_{l}^{\left(n_{l}\right)}\right\}_{l=1}^{L} ;\left\{R_{0}^{\bar{P}}\left[H_{f} ; \theta\right]\right\}_{l=1}^{L-1}\right] \\
:=\prod_{l=1}^{L}(-g)^{m_{l}+n_{l}+p_{l}+q_{l}} \chi_{\rho_{0}}\left(\gamma+\tau_{0}\right)\left(\phi_{0}(\theta) \otimes \Omega, W_{p_{1}, q_{1}}^{m_{1}, n_{1}}\left[\underline{k}_{1}^{\left(m_{1}\right)} ; \underline{\tilde{k}}_{1}{ }^{\left(n_{1}\right)}\right]\right. \\
R_{0}^{\bar{P}}\left[H_{f}+\gamma+\tau_{1} ; \theta\right] W_{p_{2}, q_{2}}^{m_{2}, n_{2}}\left[\underline{k}_{2}^{\left(m_{2}\right)} ; \underline{\tilde{k}}_{2}^{\left(n_{2}\right)}\right] \ldots \\
\left.R_{0}^{\bar{P}}\left[H_{f}+\gamma+\tau_{L-1} ; \theta\right] W_{p_{l}, q_{L}}^{m_{l}, n_{L}}\left[\underline{k}_{L}^{\left(m_{L}\right)} ; \underline{\tilde{k}}_{L}^{\left(n_{L}\right)}\right] \phi_{0}(\theta) \otimes \Omega\right) \\
\chi_{\rho_{0}}\left(\gamma+\tau_{L}\right) .
\end{gathered}
$$

We have set

$$
R_{0}^{\bar{P}}\left[H_{f} ; \theta\right]:=\bar{P}(\theta)\left[\left(H_{0}(\theta)-\xi\right) \mathbf{1}_{\operatorname{Ran}(\bar{P}(\theta))}\right]^{-1} \bar{P}(\theta)
$$

and

$$
\begin{aligned}
& W_{p_{l}, q_{l}}^{m_{l}, n_{l}}\left[\underline{k}_{l}^{\left(m_{l}\right)} ; \underline{\tilde{k}}_{l}^{\left(n_{l}\right)}\right] \\
&:=\int_{\underline{\mathbb{R}}^{3\left(p_{l}+q_{l}\right)}} G_{m_{l}+p_{l}, n_{l}+q_{l}}\left[\underline{k}_{l}^{\left(m_{l}\right)}, \underline{y}_{l}^{\left(p_{l}\right)} ; \underline{\tilde{k}}_{l}^{\left(n_{l}\right)}, \underline{\tilde{y}}_{l}^{\left(q_{l}\right)} ; \theta\right] \\
& \otimes a^{*}\left(\underline{y}_{l}^{\left(p_{l}\right)}\right) a\left(\underline{\tilde{y}}_{l}^{\left(q_{l}\right)}\right) d \underline{y}_{l}^{\left(p_{l}\right)} d \underline{\tilde{y}}_{l}^{\left(q_{l}\right)} .
\end{aligned}
$$

Besides,

$$
\tau_{l}:=\sum_{j=1}^{l}\left|\tilde{k}_{j}^{\left(n_{j}\right)}\right|+\sum_{j=l+1}^{L}\left|k_{j}^{\left(m_{j}\right)}\right|:=\sum_{j=1}^{l} \sum_{i=1}^{n_{j}}\left|\tilde{k}_{j}^{i}\right|+\sum_{j=l+1}^{L} \sum_{i=1}^{m_{j}}\left|k_{j}^{i}\right| .
$$

We come now to the proof of an estimate which is strongly related to the confinement of the center of mass imposed in our model, since it requires Assumptions $\left(\mathcal{H}_{-1 / 2}\right)$ and $\left(\mathcal{H}_{1 / 2}\right)$. It is the purpose of the following lemma:

Lemma 14. Assume that Hypotheses $\left(\mathcal{H}_{-1 / 2}\right)$ and $\left(\mathcal{H}_{1 / 2}\right)$ hold for some $\theta_{0}>0$ sufficiently small. Then there exists a positive constant $\mathrm{C}$ such that for all $\theta \in$ $D\left(0, \theta_{0}\right), \rho>0$, and $m, n, p, q \in \mathbb{N}, m+n+p+q \in\{1,2\}$,

$$
\begin{array}{r}
\left\|\left|H_{a t}+i\right|^{-\frac{1}{2} \delta_{p, 0}}\left[H_{f}+\rho\right]^{-\frac{p}{2}} W_{p, q}^{m, n}\left[\underline{k}^{(m)} ; \underline{\tilde{k}}^{(n)}\right]\left[H_{f}+\rho\right]^{-\frac{q}{2}}\left|H_{a t}+i\right|^{-\frac{1}{2} \delta_{q, 0}}\right\| \\
\leq \mathrm{C} \prod_{j=1}^{m} J_{1 / 2}\left(k_{j}\right) \prod_{j=1}^{n} J_{1 / 2}\left(\tilde{k}_{j}\right) .
\end{array}
$$


Proof. Let us distinguish between the different cases $m+n=i, p+q=j$ such that $i, j \in\{0,1,2\}$ and $i+j \in\{1,2\}$. First, if $m+n=0$ and $p+q \in\{1,2\}$, since Hypothesis $\left(\mathcal{H}_{-1 / 2}\right)$ is satisfied, Lemma 9 implies

$$
\left\|\left|H_{a t}+i\right|^{-\frac{1}{2} \delta_{p, 0}}\left[H_{f}+\rho\right]^{-p / 2} W_{p, q}^{0,0}\left[H_{f}+\rho\right]^{-q / 2}\left|H_{a t}+i\right|^{-\frac{1}{2} \delta_{q, 0}}\right\| \leq \mathrm{C} .
$$

Suppose now that $m+n \in\{1,2\}$ and $p+q=0$. Then Hypothesis $\left(\mathcal{H}_{1 / 2}\right)$ leads to

$$
\left\|\left|H_{a t}+i\right|^{-\frac{1}{2}} W_{0,0}^{m, n}\left[\underline{k}^{(m)} ; \underline{\tilde{k}}^{(n)}\right]\left|H_{a t}+i\right|^{-\frac{1}{2}}\right\| \leq \mathrm{C} \prod_{j=1}^{m} J_{1 / 2}\left(k_{j}\right) \prod_{j=1}^{n} J_{1 / 2}\left(\tilde{k}_{j}\right) .
$$

Finally, we have to deal with the possibility $m+n=1$ and $p+q=1$. Let us assume, for instance, that $m=q=1$ and $n=p=0$; the other cases could be treated in the same way. We have:

$$
\begin{aligned}
\left\|\left|H_{a t}+i\right|^{-\frac{1}{2}} W_{0,1}^{1,0}[\underline{k}]\left[H_{f}+\rho\right]^{-\frac{1}{2}}\right\| \\
=\left\|\int_{\mathbb{R}^{3}}\left|H_{a t}+i\right|^{-\frac{1}{2}} G_{1,1}[\underline{k} ; \underline{y} ; \theta] \otimes a(\underline{y}) d \underline{y}\left[H_{f}+\rho\right]^{-\frac{1}{2}}\right\| \\
\leq\left[\int_{\mathbb{R}^{3}}\left\|\frac{1}{|y|}\left|H_{a t}+i\right|^{-\frac{1}{2}} G_{1,1}[\underline{k} ; \underline{y} ; \theta]\right\|^{2} d \underline{y}\right]^{\frac{1}{2}} \\
\quad \times \sup _{\|\Psi\|=1}\left[\int_{\mathbb{R}^{3}}|y|\left\|\left(H_{f}+|y|\right)^{-1 / 2} a(\underline{y}) \Psi\right\|^{2} d \underline{y}\right]^{\frac{1}{2}} .
\end{aligned}
$$

One can see that the second term in the last line is bounded by a constant. The first term is estimated using Hypothesis $\left(\mathcal{H}_{1 / 2}\right)$, which yields

$$
\left\|\left|H_{a t}+i\right|^{-\frac{1}{2}} W_{0,1}^{1,0}[\underline{k}]\left[H_{f}+\rho\right]^{-\frac{1}{2}}\right\| \leq \mathrm{C} J_{1 / 2}(k) .
$$

As the other cases would follow similarly, the proof of the lemma is complete.

\section{Back to the proof of Theorem 13}

To finish the proof of Theorem 13, with the help of Lemma 14, it suffices to follow [4], with some modifications. Namely, inserting into (105) the identity $\left[H_{f}+\right.$ $\left.\rho_{0}\right] \phi_{0}(\theta) \otimes \Omega=\rho_{0} \phi_{0}(\theta) \otimes \Omega$ together with the estimates obtained in Lemmas 10 and 14 , we get in a way similar to [4, Lemma 3.7]:

$$
\begin{aligned}
& \left|\widetilde{D}_{L}\left[\xi ; \gamma ;\left\{W_{p_{l}, q_{l}}^{m_{l}, n_{l}} ; \underline{k}_{l}^{\left(m_{l}\right)} ; \underline{\tilde{k}}_{l}^{\left(n_{l}\right)}\right\}_{l=1}^{L} ;\left\{R_{0}^{\bar{P}}\left[H_{f} ; \theta\right]\right\}_{l=1}^{L-1}\right]\right| \\
& \leq \prod_{l=1}^{L}\left(\mathrm{C}_{1} g \rho_{0}{ }^{-1 / 2}\right)^{m_{l}+n_{l}+p_{l}+q_{l}} \rho_{0}{ }^{1-\frac{1}{2}(M+N)} \mathrm{C}_{2}^{M+N} \prod_{j=1}^{M} J_{1 / 2}\left(k_{j}\right) \prod_{j=1}^{N} J_{1 / 2}\left(\tilde{k}_{j}\right) .
\end{aligned}
$$


Here, $\mathrm{C}_{1}, \mathrm{C}_{2}$ denote positive real numbers depending respectively on $\nu, \Lambda$ and $\Lambda$. Inserting (113) into (103) leads to

$$
\begin{aligned}
& \left|\widetilde{w}_{M, N}^{\mathrm{eff}}\left[\xi ; \gamma ; \underline{k}^{(M)} ; \underline{\tilde{k}}^{(N)}\right]\right| \\
& \leq \rho_{0}{ }^{1-\frac{1}{2}(M+N)}\left(\mathrm{C}_{3} g \rho_{0}{ }^{-1 / 2}\right)^{M+N+2 \delta_{M+N, 0}} \prod_{j=1}^{M} J_{1 / 2}\left(k_{j}\right) \prod_{j=1}^{N} J_{1 / 2}\left(\tilde{k}_{j}\right)
\end{aligned}
$$

for some positive constant $\mathrm{C}_{3}$. Next, regarding the definition of the Banach space of Hamiltonians at the beginning of this subsection, we have to consider the derivative of $\widetilde{D}_{L}[\ldots]$ with respect to $\gamma$. To this end, note that

$$
\begin{aligned}
R_{0}^{\bar{P}}\left[H_{f} ; \theta\right]= & {\left[\bar{P}_{a t}(\theta) H_{a t}(\theta) \otimes \mathbf{1}+e^{-i \nu} \bar{P}_{a t}(\theta) \otimes\left(H_{f}-\xi\right)\right]^{-1} \bar{P}_{a t}(\theta) } \\
& +P_{a t}(\theta) \otimes \frac{\bar{\chi}_{\rho_{0}}\left(H_{f}\right)^{2}}{e^{-i \nu} H_{f}-\xi}
\end{aligned}
$$

which yields

$$
\partial_{H_{f}} R_{0}^{\bar{P}}\left[H_{f} ; \theta\right]=-e^{-i \nu} R_{0}^{\bar{P}}\left[H_{f} ; \theta\right]^{2}+P_{a t}(\theta) \otimes \frac{2 \bar{\chi}_{\rho_{0}}\left(H_{f}\right) \partial_{H_{f}} \bar{\chi}_{\rho_{0}}\left(H_{f}\right)}{e^{-i \nu} H_{f}-\xi} .
$$

Using this together with Leibniz' rule, Lemma 10 and Lemma 14, we obtain as in (113):

$$
\begin{aligned}
& \left|\partial_{\gamma} \widetilde{D}_{L}\left[\xi ; \gamma ;\left\{W_{p_{l}, q_{l}}^{m_{l}, n_{l}} ; \underline{k}_{l}^{\left(m_{l}\right)} ; \underline{\tilde{k}}_{l}^{\left(n_{l}\right)}\right\}_{l=1}^{L} ;\left\{R_{0}^{\bar{P}}\left[H_{f} ; \theta\right]\right\}_{l=1}^{L-1}\right]\right| \\
& \leq \prod_{l=1}^{L}\left(\mathrm{C}_{1} g \rho_{0}^{-1 / 2}\right)^{m_{l}+n_{l}+p_{l}+q_{l}} \rho_{0}^{-\frac{1}{2}(M+N)} \mathrm{C}_{2}^{M+N} \prod_{j=1}^{M} J_{1 / 2}\left(k_{j}\right) \prod_{j=1}^{N} J_{1 / 2}\left(\tilde{k}_{j}\right),
\end{aligned}
$$

provided that $\mathrm{C}_{1}, \mathrm{C}_{2}$ are chosen sufficiently large. Hence, inserting (117) into (103), we get

$$
\begin{aligned}
& \left|\partial_{\gamma} \widetilde{w}_{M, N}^{\mathrm{eff}}\left[\xi ; \gamma ; \underline{k}^{(M)} ; \underline{\tilde{k}}^{(N)}\right]\right| \\
& \leq \rho_{0}{ }^{-\frac{1}{2}(M+N)}\left(\mathrm{C}_{3} g \rho_{0}{ }^{-1 / 2}\right)^{M+N+2 \delta_{M+N, 0}} \prod_{j=1}^{M} J_{1 / 2}\left(k_{j}\right) \prod_{j=1}^{N} J_{1 / 2}\left(\tilde{k}_{j}\right) .
\end{aligned}
$$

To conclude, we come back to the operator $H_{(0)}[z]$ defined in $(96)-(98)$. The identity

$$
H_{(0)}[z]=\frac{e^{i \nu}}{\rho_{0}} \mathcal{U}_{\rho_{0}} \widetilde{H}_{\mathrm{eff}}\left[Z_{(0)}^{-1}(z)\right] \mathcal{U}_{\rho_{0}}^{*}
$$

together with (101)-(102) implies that for any $z \in D_{1 / 2}$ :

$$
H_{(0)}[z]=\mathbf{1}_{H_{f}<1}\left[E_{(0)}[z]+T_{(0)}\left[z ; H_{f}\right]+W_{(0)}\left[z ; H_{f}\right]\right] \mathbf{1}_{H_{f}<1},
$$


with

$$
\begin{aligned}
E_{(0)}[z] & :=w_{0,0}^{(0)}[z, 0], \\
T_{(0)}\left[z ; H_{f}\right] & :=H_{f}+w_{0,0}^{(0)}\left[z ; H_{f}\right]-w_{0,0}^{(0)}[z, 0], \\
W_{(0)}\left[z ; H_{f}\right] & :=\sum_{M+N \geq 1} W_{M, N}^{(0)}\left[z ; H_{f}\right],
\end{aligned}
$$

and

$$
W_{M, N}^{(0)}\left[z ; H_{f}\right]:=\int_{\underline{\mathbb{R}}^{3(M+N)}} a^{*}\left(\underline{k}^{(M)}\right) w_{M, N}^{(0)}\left[z ; H_{f} ; \underline{k}^{(M)} ; \underline{\tilde{k}}^{(N)}\right] a\left(\underline{\tilde{k}}^{(N)}\right) d \underline{k}^{(M)} d \underline{\tilde{k}}^{(N)} .
$$

Furthermore, for all $M+N \geq 0, w_{M, N}^{(0)}$ is related to $\widetilde{w}_{M, N}^{\mathrm{eff}}$ through

$$
w_{M, N}^{(0)}\left[z ; \gamma ; \underline{k}^{(M)} ; \underline{\tilde{k}}^{(N)}\right]=\rho_{0}^{\frac{3}{2}(M+N)-1} \widetilde{w}_{M, N}^{\mathrm{eff}}\left[Z_{(0)}^{-1}(z) ; \rho_{0} \gamma ; \rho_{0} \underline{\underline{k}}^{(M)} ; \rho_{0} \underline{\tilde{k}}^{(N)}\right] .
$$

Thus, (114) and (118) yield

$$
\begin{aligned}
& \left|w_{M, N}^{(0)}\left[z ; \gamma ; \underline{k}^{(M)} ; \underline{\tilde{k}}^{(N)}\right]\right|+\left|\partial_{\gamma} w_{M, N}^{(0)}\left[z ; \gamma ; \underline{k}^{(M)} ; \underline{\tilde{k}}^{(N)}\right]\right| \\
& \leq 2 \rho_{0}{ }^{(M+N)}\left(\mathrm{C}_{3} g \rho_{0}{ }^{-1 / 2}\right)^{M+N+2 \delta_{M+N, 0}} \prod_{j=1}^{M} J_{1 / 2}\left(\rho_{0} k_{j}\right) \prod_{j=1}^{N} J_{1 / 2}\left(\rho_{0} \tilde{k}_{j}\right) \\
& \leq \rho_{0}{ }^{\frac{3}{2}(M+N)}\left(\mathrm{C}_{4} g \rho_{0}{ }^{-1 / 2}\right)^{M+N+2 \delta_{M+N, 0}} \prod_{j=1}^{M}\left|k_{j}\right|^{1 / 2} \prod_{j=1}^{N}\left|\tilde{k}_{j}\right|^{1 / 2}
\end{aligned}
$$

where $\mathrm{C}_{4}$ denotes a positive real number (depending on $\nu$ and $\Lambda$ ). In other words, $H_{(0)}[z]=H\left(\underline{w}_{(0)}[z]\right)$ with $\underline{w}_{(0)}=\left(E_{(0)}, T_{(0)},\left(w_{M, N}^{(0)}\right)_{M+N \geq 1}\right)$ and

$$
\begin{aligned}
\sup _{z \in D_{1 / 2}}\left|E_{(0)}[z]\right| & \leq \mathrm{C}_{4} g^{2} \rho_{0}^{-1}, \\
\sup _{z \in D_{1 / 2}}\left\|T_{(0)}[z ; \gamma]-\gamma\right\|_{\mathcal{T}} & \leq 2 \mathrm{C}_{4} g^{2} \rho_{0}^{-1}, \\
\sup _{z \in D_{1 / / 2}}\left\|\left(w_{M, N}^{(0)}[z]\right)_{M+N \geq 1}\right\|_{\zeta}^{\#} & \leq \sum_{M+N \geq 1}\left(\mathrm{C}_{4} g \rho_{0}{ }^{-1 / 2}\right)^{M+N}=\frac{\mathrm{C}_{4} g \rho_{0}{ }^{-1 / 2}}{\left(1-\mathrm{C}_{4} g \rho_{0}-1 / 2\right)},
\end{aligned}
$$

with $\zeta<1$ chosen so that $\zeta \geq \rho_{0}^{1 / 2}$, and provided that $\mathrm{C}_{4} g \rho_{0}^{-1 / 2}<1$. Hence, the proof of the theorem is complete, except for the fact that $H_{(0)}[z]$ is analytic on $D_{1 / 2}$. But since $\partial_{\xi} R_{0}^{\bar{P}}\left[H_{f} ; \theta\right]=-R_{0}^{\bar{P}}\left[H_{f} ; \theta\right]^{2}$, one can see that $\partial_{z} w_{M, N}^{(0)}$ $\left[z ; \gamma ; \underline{k}^{(M)} ; \underline{\tilde{k}}^{(N)}\right]$ is bounded in a way similar to (118), which gives the analyticity of $H_{(0)}[z]$.

To sum up, we have showed that, for any $\zeta<1$ such that $\zeta \geq \rho_{0}^{1 / 2}, H_{(0)}[\cdot]$ belongs to $H(B(\rho / 8, \rho / 8))$ for any $\rho>0$, provided that $\rho_{0}$ is less than $\left(\delta_{j} \sin \nu\right) / 2$ 
and that $g \rho_{0}{ }^{-1 / 2}$ is sufficiently small. Moreover, by [11], $H_{(0)}[\cdot]$ is isospectral to the initial Hamiltonian $H_{g}(\theta)$, in the sense that

$$
z \in \sigma\left(H_{(0)}[z]\right) \cap D_{1 / 2} \Leftrightarrow Z_{(0)}^{-1}(z) \in \sigma\left(H_{g}(\theta)\right) \cap D_{\rho_{0} / 2} .
$$

Now, we appeal to the results proved in [4] and [2]: the renormalization transformation $\mathcal{R}_{\rho}$ is defined for any $\underline{w}=\left(E, T,\left(w_{M, N}\right)_{M+N \geq 1}\right)$ in $B(\rho / 8, \rho / 8)$ by

$$
\begin{aligned}
\mathcal{R}_{\rho}(H(\underline{w}[z]))-z:=\rho^{-1} \mathcal{U}_{\rho} \mathcal{F}_{\chi_{\rho}\left(H_{f}\right)}\left[H\left(\underline{w}\left[Z^{-1}(z)\right]\right)-Z^{-1}(z),\right. \\
\left.E\left[Z^{-1}(z)\right]+T\left[Z^{-1}(z) ; H_{f}\right]-Z^{-1}(z)\right] \mathcal{U}_{\rho}^{*},
\end{aligned}
$$

where the map $Z$ is a bijection defined by

$$
Z:\{\xi,|\xi-E[\xi]| \leq \rho / 2\} \rightarrow D_{1 / 2}, \quad \xi \mapsto \rho^{-1}(\xi-E[\xi]) .
$$

Then we have (see [2]):

Theorem 15. Fix $\rho:=\left(16 \mathrm{C}_{\Theta}\right)^{-2}$ and $\zeta:=\left(4 \mathrm{C}_{\Theta}\right)^{-1} \rho^{1 / 2}$ where $\mathrm{C}_{\Theta} \geq 1$ is a constant only depending on the smooth function $\Theta$ defined in (65). Then,

$$
\mathcal{R}_{\rho}: H(B(\beta, \varepsilon)) \rightarrow H\left(B\left(\beta+\frac{\varepsilon}{2}, \frac{\varepsilon}{2}\right)\right),
$$

for any $0<\beta \leq \varepsilon_{0}, 0<\varepsilon \leq \varepsilon_{0}$, where $\varepsilon_{0}:=\left(8 \mathrm{C}_{\Theta}\right)^{-1} \rho$.

Proof. Recall that the Banach space $\mathcal{W}_{\geq 0}$ that we have chosen in this paper is different from the one defined in [2]; indeed, we have used a supremum in the definition of the norm (79), where the authors used a $\mathrm{L}^{2}$-norm in [2]. This modification allows to simplify some estimates, but it also requires to modify the proof of this theorem. However, the modifications are straightforward and we do not reproduce the proof.

To conclude, we state a result which provides the existence of resonances for the Hamiltonian $\widetilde{H}_{U}^{V}$; its proof can be found in $[2,4]$. We define for $n \geq 1$

$$
H_{(n)}[\cdot]:=H\left(\left(E_{(n)}, T_{(n)},\left(w_{M, N}^{(n)}\right)_{M+N \geq 1}\right)\right):=\mathcal{R}_{\rho}^{n}\left(H_{(0)}[\cdot]\right)
$$

and, as in (128), $Z_{(n)}$ is a bijection defined by

$$
Z_{(n)}:\left\{z \in D_{1 / 2},\left|z-E_{(n-1)}[z]\right| \leq \rho / 2\right\} \rightarrow D_{1 / 2}, \quad z \mapsto \frac{1}{\rho}\left(z-E_{(n-1)}[z]\right) .
$$

Notice that, choosing $\rho, \zeta, \varepsilon_{0}, \beta, \varepsilon$ as in Theorem 15 and $\rho_{0}$ such that $0<\rho_{0} \leq$ $\min \left(\zeta^{2},\left(\delta_{j} \sin \nu\right) / 2\right)$, we obtain from Theorems 13 and 15 :

$$
H_{(n)}[\cdot] \in H\left(B\left(\beta+\varepsilon, \frac{\varepsilon}{2^{n}}\right)\right),
$$

for any $n \geq 0$, provided that $g \rho_{0}^{-1 / 2}$ is sufficiently small. Then it is proved that: 
Theorem 16. Let $g>0$ and $\rho_{0}>0$ sufficiently small. Assume that Hypotheses $\left(\mathcal{H}_{\text {an }}\right),\left(\mathcal{H}_{-1 / 2}\right)$ and $\left(\mathcal{H}_{1 / 2}\right)$ hold for some $\theta_{0}$ sufficiently small. Fix $\rho$ and $\zeta$ as in Theorem 15. Then, for all $\theta=i \nu \in D\left(0, \theta_{0}\right)$, where $\nu>0$ is chosen such that $\rho_{0} \leq\left(\delta_{j} \sin \nu\right) / 2<1$, the spectrum of $H_{g}(\theta)$ is located as follows:

$$
\sigma\left(H_{g}(\theta)\right) \cap D_{\rho_{0} / 2} \subset E_{(\infty)}+K_{(\infty)} .
$$

Here, $E_{(\infty)}:=\lim _{n \rightarrow \infty} Z_{(0)}^{-1} \circ Z_{(1)}^{-1} \circ \cdots \circ Z_{(n)}^{-1}(0)$ is a simple eigenvalue of $H_{g}(\theta)$, and $K_{(\infty)}$ is a complex domain defined by

$$
K_{(\infty)}:=\left\{e^{-i \nu} a+b, 0 \leq a \leq 1,|b| \leq \mathrm{Ca}^{\tau}\right\},
$$

where $\tau>1$ and where $\mathrm{C}$ is a positive constant which can be chosen strictly less than 1 provided that $g$ is sufficiently small. Assuming moreover that $\left(\mathcal{H}_{\Gamma_{j}}\right)$ is fulfilled, this implies that $E_{(\infty)}$ is a resonance for $H_{g}$.

\section{Appendix A. Proof of a hypothesis related to the Fermi Golden Rule}

In this appendix, we prove that, for suitably chosen parameters, the assumption $\left(\mathcal{H}_{\Gamma_{1}}\right)$ (see Subsection 3.1) related to the Fermi Golden Rule is satisfied in the case of the confined hydrogen atom $H_{U}^{V}$. Recall that $\left\{E_{l}\right\}$ denotes the sequence of the eigenvalues of $p^{2} / 2 \mu+V$ and that $\left\{e_{n}\right\}$ denotes the sequence of the eigenvalues of $P^{2} / 2 M+U$, where $U(R)=\beta^{2} R^{2}+\mathrm{c}_{0}$. Note that $e_{1}-e_{0}=\sqrt{2 / M} \beta$, whereas $E_{1}-E_{0}=O(1)$. Thus, for $\beta$ sufficiently small, $E_{0}+e_{1}$ is the first eigenvalue (with multiplicity 3 ) above the bottom of the spectrum $E_{0}+e_{0}$ of $H_{a t}$. We have to show that

$$
\Gamma_{1}=\min \left\{\sigma\left(\operatorname{Im}\left(Z_{1}^{\text {od }}\right)\right)\right\}
$$

is strictly positive, where $Z_{1}^{\text {od }}$ is the matrix

$$
Z_{1}^{\text {od }}=\int_{\underline{\mathbb{R}}^{3}} P_{a t, 1} G_{0,1}(\underline{k}) \bar{P}_{a t, 1}\left[H_{a t}-\left(E_{0}+e_{1}\right)+|k|-i 0\right]^{-1} \bar{P}_{a t, 1} G_{1,0}(\underline{k}) P_{a t, 1} d \underline{k} .
$$

Here $P_{a t, 1}$ denotes the projection onto the eigenspace of $H_{a t}$ associated with $E_{0}+$ $e_{1}$, and $\bar{P}_{a t, 1}=1-P_{a t, 1}$. We choose explicit polarization vectors:

$$
\varepsilon_{1}(k):=\frac{\left(k_{2},-k_{1}, 0\right)}{\sqrt{k_{1}^{2}+k_{2}^{2}}}, \quad \varepsilon_{2}(k):=\frac{k}{|k|} \wedge \varepsilon_{1}(k),
$$

where $k=\left(k_{1}, k_{2}, k_{3}\right)$. Recall that $q^{2}=g^{2 / 3} / \Lambda$.

Proposition 17. Let $0<q^{2} \ll \beta^{5 / 2} \ll 1$. Then $\left(\mathcal{H}_{\Gamma_{1}}\right)$ is satisfied, with

$$
\Gamma_{1}=\mathrm{C} \beta^{5 / 2}+O\left(q^{2}\right),
$$

where $\mathrm{C}$ is a positive constant. 
Proof. Let $\Psi_{0}$ be a non-degenerate ground state of $p^{2} / 2 \mu+V$. Let $\phi_{0}$ and $\phi_{1}$ be first two normalized eigenstates associated with the 1-dimensional harmonic oscillator $d^{2} / d x^{2}+\beta^{2} x^{2}$ :

$$
\phi_{0}(x)=\left(\frac{\sqrt{2 M} \beta}{\pi}\right)^{1 / 4} e^{-\sqrt{2 M} \beta x^{2} / 2}, \quad \phi_{1}(x)=\frac{(\sqrt{2 M} \beta)^{3 / 4}}{\pi^{1 / 4}} x e^{-\sqrt{2 M} \beta x^{2} / 2} .
$$

Then we can write the eigenstates $\Phi_{0}$ and $\Phi_{1}^{j}$ (for $j=1,2,3$ ) associated respectively with the eigenvalues $e_{0}$ and $e_{1}$ of $P^{2} / 2 M+U$ as

$$
\Phi_{0}(R)=\prod_{j=1,2,3} \phi_{0}\left(R_{j}\right), \quad \Phi_{1}^{j}(R)=\left(\prod_{i=1,2,3, i \neq j} \phi_{0}\left(R_{i}\right)\right) \phi_{1}\left(R_{j}\right),
$$

with $R=\left(R_{1}, R_{2}, R_{3}\right)$. By (136), we have to compute for $i, j \in\{1,2,3\}$ :

$$
\begin{aligned}
\left(\operatorname{Im}\left(Z_{1}^{\text {od }}\right)\right)_{i, j}= & \int_{\underline{\mathbb{R}}^{3}}\left[\int_{\mathbb{R}^{6}} \Psi_{0}(r) \Phi_{1}^{i}(R) G_{0,1}(\underline{k}) \Psi_{0}(r) \Phi_{0}(R) d r d R\right] \\
& \times \mathcal{C}\left[\int_{\mathbb{R}^{6}} \Psi_{0}(r) \Phi_{1}^{j}(R) G_{0,1}(\underline{k}) \Psi_{0}(r) \Phi_{0}(R) d r d R\right] \\
& \times \delta\left(|k|-\left(e_{1}-e_{0}\right)\right) d \underline{k},
\end{aligned}
$$

where $\mathcal{C}$ denotes the complex conjugation in $\mathbb{C}$. Recall that

$$
\begin{aligned}
G_{0,1}(\underline{k})= & \frac{-i Z}{2 m_{1} \Lambda^{3 / 2}} \frac{\widehat{\chi}_{\Lambda}(k)}{2 \pi \sqrt{|k|}} e^{i Z q^{2} k \cdot x_{1}} \varepsilon_{\lambda}(k) \cdot \nabla_{x_{1}} \\
& +\frac{i Z^{2}}{2 m_{2} \Lambda^{3 / 2}} \frac{\widehat{\chi}_{\Lambda}(k)}{2 \pi \sqrt{|k|}} e^{i Z q^{2} k \cdot x_{2}} \varepsilon_{\lambda}(k) \cdot \nabla_{x_{2}} .
\end{aligned}
$$

We denote by $A$ the matrix obtained from $\operatorname{Im}\left(Z_{1}^{\text {od }}\right)$ by replacing $e^{i Z q^{2} k \cdot x_{j}}$ by 1 in the definition of $G_{0,1}(\underline{k})$. Let us show that $A$ is diagonal and positive. In the spherical coordinates defined for $\rho \geq 0,0 \leq \theta \leq 2 \pi$ and $0 \leq \varphi \leq \pi$ by

$$
k_{1}=\rho \cos \theta \sin \varphi, \quad k_{2}=\rho \sin \theta \sin \varphi, \quad k_{3}=\rho \cos \varphi,
$$

the polarization vectors (137) become

$$
\varepsilon_{1}(k)=(\sin \theta,-\cos \theta, 0), \quad \varepsilon_{2}(k)=(\cos \theta \cos \varphi, \sin \theta \cos \varphi,-\sin \varphi) .
$$

Let us compute, for instance, $A_{1,2}$ : we have

$$
\begin{aligned}
A_{1,2}= & \mathrm{C}_{0} \frac{\beta^{2} \widehat{\chi}_{\Lambda}(\sqrt{2 / M} \beta)}{\sqrt{\beta}} \\
& \times\left[\int_{0}^{2 \pi} \int_{0}^{\pi}\left(\phi_{1},(\sin \theta) \phi_{0}^{\prime}\right) \times\left(\phi_{1},(-\cos \theta) \phi_{0}^{\prime}\right) \sin \varphi d \varphi d \theta\right. \\
& \left.+\int_{0}^{2 \pi} \int_{0}^{\pi}\left(\phi_{1},(\cos \theta \cos \varphi) \phi_{0}^{\prime}\right) \times\left(\phi_{1},(\sin \theta \cos \varphi) \phi_{0}^{\prime}\right) \sin \varphi d \varphi d \theta\right]
\end{aligned}
$$


where $\mathrm{C}_{0}$ is a positive constant and where $\phi_{0}^{\prime}:=d \phi_{0} / d x$. Integrating over $\theta$ yields $A_{1,2}=0$. The same would hold for $A_{i, j}$ with $i \neq j$, as follows by integrating over $\theta$ or $\varphi$. Now, let us compute, for instance, the diagonal coefficient $A_{1,1}$ : we have

$$
\begin{aligned}
A_{1,1}= & \mathrm{C}_{1} \frac{\beta^{2} \widehat{\chi}_{\Lambda}(\sqrt{2 / M} \beta)}{\sqrt{\beta}} \\
& \times\left[\int_{0}^{2 \pi} \int_{0}^{\pi}\left(\phi_{1},(\sin \theta) \phi_{0}^{\prime}\right)^{2} \sin \varphi d \varphi d \theta\right. \\
& \left.+\int_{0}^{2 \pi} \int_{0}^{\pi}\left(\phi_{1},(\cos \theta \cos \varphi) \phi_{0}^{\prime}\right)^{2} \sin \varphi d \varphi d \theta\right],
\end{aligned}
$$

where $\mathrm{C}_{1}$ a positive constant. The second term in the previous sum vanishes, whereas the first one gives:

$$
\int_{0}^{2 \pi} \int_{0}^{\pi}\left(\phi_{1},(\sin \theta) \phi_{0}^{\prime}\right)^{2} \sin \varphi d \varphi d \theta=c_{1}\left(\phi_{1}, \phi_{0}^{\prime}\right)^{2}=c_{1}^{\prime} \beta,
$$

where $c_{1}$ and $c_{1}^{\prime}$ are positive constants. Thus

$$
A_{1,1}=\mathrm{C}_{1}^{\prime} \widehat{\chi}_{\Lambda}(\sqrt{2 / M} \beta) \beta^{5 / 2},
$$

where $\mathrm{C}_{1}^{\prime}$ is a positive constant. Likewise one could show that

$$
A_{2,2}=\mathrm{C}_{2}^{\prime} \widehat{\chi}_{\Lambda}(\sqrt{2 / M} \beta) \beta^{5 / 2}, \quad A_{3,3}=\mathrm{C}_{3}^{\prime} \widehat{\chi}_{\Lambda}(\sqrt{2 / M} \beta) \beta^{5 / 2},
$$

where $\mathrm{C}_{2}^{\prime}$ and $\mathrm{C}_{3}^{\prime}$ are positive constants. To finish the proof, we have to check that $\operatorname{Im}\left(Z_{1}^{\text {od }}\right)-A$ is small compared to $A$. But the matrix $\operatorname{Im}\left(Z_{1}^{\text {od }}\right)-A$ is $\operatorname{Im}\left(Z_{1}^{\text {od }}\right)$ where, in at least one of the two operators $G_{0,1}(k, 1), G_{0,1}(k, 2)$, the terms $e^{i Z q^{2} k \cdot x_{j}}$ are replaced by $e^{i Z q^{2} k \cdot x_{j}}-1$. Hence, using that

$$
\left|e^{i Z q^{2} k \cdot x_{j}}-1\right| \leq Z q^{2}|k|\left|x_{j}\right|
$$

one can see that for $i, j \in\{1,2,3\}$ :

$$
\left|\left(\operatorname{Im}\left(Z_{1}^{\text {od }}\right)-A\right)_{i, j}\right| \leq \mathrm{C}(\beta) q^{2},
$$

where $\mathrm{C}(\beta)$ is a bounded positive constant depending on $\beta$. Provided that $q^{2}$ is sufficiently small, this implies that $\Gamma_{1}>0$ and the proof is complete.

Remark 18. Let $j$ be such that $E_{1}+e_{0}$ is the jth eigenvalue of $H_{a t}$. Using arguments similar to the ones of the proof of Proposition 17, under the same assumptions, one could show that $\Gamma_{j}>0$, with $\Gamma_{j} \gg \beta$ provided that $\beta$ is chosen sufficiently small. Thus, we see that the first eigenvalues $E_{0}+e_{n}$ (with $n$ small) lead to resonances that should be much closer to the real axis than the other ones coming from the eigenvalues of the form $E_{l}+e_{n}$ with $l \geq 1$. In some sense, this is related to the Lamb-Dicke effect mentioned in our introduction. 


\section{References}

[1] L. Amour and J. Faupin, The confined hydrogenoïd ion in non-relativistic quantum electrodynamics, Cubo Math. J. 9(2) (2007), 103-137.

[2] V. Bach, T. Chen, J. Fröhlich and I. M. Sigal, Smooth Feshbach map and operatortheoretic renormalization group methods, J. Funct. Anal. 203 (2002), 44-92.

[3] V. Bach, J. Fröhlich and I. M. Sigal, Quantum electrodynamics of confined nonrelativistic particles, Adv. in Math. 137 (1998), 299-395.

[4] V. Bach, J. Fröhlich and I. M. Sigal, Renormalization group analysis of spectral problems in quantum field theory, Adv. in Math. 137 (1998), 205-298.

[5] V. Bach, J. Fröhlich and I. M. Sigal, Spectral analysis for systems of atoms and molecules coupled to the quantized radiation fields, Comm. Math. Phys. 207(2) (1999), 249-290.

[6] C. Cohen-Tannoudji, Cours au collège de France 2003-2004, www.phys.ens/cours/ college-de-france.

[7] C. Cohen-Tannoudji, J. Dupont-Roc and G. Grynberg, Processus d'interaction entre photons et atomes (Edition du CNRS, 2001).

[8] C. Cohen-Tannoudji, J. Dupont-Roc and G. Grynberg, Photons et atomes, (Edition du CNRS, 2001).

[9] R. H. Dicke, The effect of collisions upon the Doppler width of spectral lines, Phys. Rev. 89(2) (1953), 472-473.

[10] A. Fehli, Analyse spectrale de l'hamiltonien associé à un atome non relativiste couplé à un champ électromagnétique quantifié, Thèse de l'université Paris 13, (2002).

[11] M. Griesemer and D. Hasler, On the smooth Feshbach-Schur map, Preprint mp-arc, 07-102, (2007).

[12] S. Gustafson and I.M. Sigal, Mathematical concepts of quantum mechanics, (Springer-Verlag, 2003).

[13] T. Kato, Perturbation theory for linear operators, (Springer-Verlag, 1966).

[14] M. Reed and B. Simon, Methods of modern mathematical physics, vol. IV, Analysis of operators, (Academic Press, New York, 1978).

[15] H. Spohn, Dynamics of charged particles and their radiation field, (Cambridge University press, 2004).

[16] T. Taïeb, R. Dum, J. I. Cirac, P. Marte and P. Zoller, Cooling of atoms in laserinduced potential wells, Phys. Rev. A 49 (1994), 4876-4887.

[17] D. J. Wineland and W. M. Itano, Laser cooling of atoms, Phys. Rev. A 20(4) (1979), $1521-1540$.

Jérémy Faupin

Laboratoire de Mathématiques EDPPM, UMR-CNRS 6056, Université de Reims

Moulin de la Housse - BP 1039

F-51687 Reims Cedex 2, France

e-mail: jeremy.faupin@univ-reims.fr

Communicated by Vincent Rivasseau.

Submitted: October 19, 2007.

Accepted: January 22, 2008. 